\title{
Hydride transfer reactions of 5-(2-alkohybenzylidene) barbituric acids: Synthesis of 2,4,6-trioxoperhydropyrimidine-5-spiro-3'- chromanes
}

\author{
Konstantin A. Krasnov, ${ }^{\text {a* }}$ Pavel V. Dorovatovskii, ${ }^{b}$ Yan V. Zubavichus, ${ }^{\text {b }}$ \\ Tatiana V. Timofeeva ${ }^{c}$ and Victor N. Khrustalev ${ }^{\mathrm{d}, \mathrm{e} *}$ \\ ${ }^{a}$ Institute of Toxicology, St. Petersburg, Russia \\ E-mail: krasnov_tox@mail.ru \\ ${ }^{b}$ National Research Center "Kurchatov Institute”, Moscow, Russia \\ ${ }^{c}$ New Mexico Highlands University, Las Vegas, NM USA \\ 'Peoples' Friendship University of Russia (RUDN), Moscow, Russia \\ E-mail:vnkhrustalev@gmail.com \\ ${ }^{e}$ Nesmeyanov Institute of Organoelement Compounds of RAS, Moscow, Russia \\ E-mail: vkh@xray.ineos.ac.ru
}<smiles>[R2]c1cccc(C=C2C(=O)N([R7])C(=O)N([Z1])C2=O)c1OC([R])([Z1])[2H]</smiles>

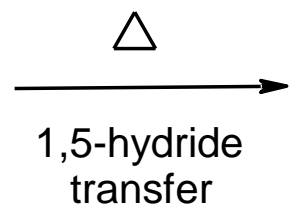

\section{ARTICLE INFO}

Article history:

Received

Received in revised form

Accepted

Available online

Keywords:

Keyword_1 5-(2-Alkoxyphenylmethylene)barbituric acids

Keyword_2 1,5-Hydride shift

Keyword_3 Thermal isomerization

Keyword_4 T-reaction

Keyword_5 Trioxoperhydropyrimidine-5-

spiro-3'-chromane

\section{ABSTRACT}

The thermal cyclization of 5-(2-phenoxymethylphenylmethylene)barbituric acid and its derivatives affords 2,4,6-trioxoperhydropyrimidine-5-spiro-3'-chromanes.

The reactions require no catalysts and proceed at temperatures from 118 to $240{ }^{\circ} \mathrm{C}$ depending on the substrate activity. These cyclization reactions are analogous to T-reactions of tertiary amines involving the hydride transfer. 


\section{Introduction}

Barbituric acid derivatives attract considerable interest because of their pharmaceutical importance. A number of 5-alkyl- and aryl barbiturates are used as hypnotic, sedative, anticonvulsant and antihypertensive drugs. ${ }^{1,2}$ Many other types of biological activities were also reported for this class of compounds. ${ }^{3}$

Furthermore, barbituric acids can be regarded as multifunctional building blocks with interesting and non-trivial chemistry ${ }^{3,4}$, which are often used as synthones in the rational design of diverse fused pyrimidine derivatives including synthetic analogs of a naturally occurring compounds as well as other systems with promising biological properties. ${ }^{5,6}$

In this regard the chemistry of barbituric acids experiences rapid development and its toolbox is being constantly enriched with new unexpected reactions. Among such reactions, the recently reported radical-radical cyclizations of barbiturates triggered by electron-transfer reduction by $\mathrm{SmI}_{2}{ }^{7}$, and the tert-amino-effect reactions that proceeds by a hydride-transfer mechanism ${ }^{8}$ are particular note.

Recently, a series of unusual conversions of ortho-vinylaryl alkyl ethers to dihydrobenzopyran (chromane) derivatives via a hydride transfer has been reported ${ }^{9,10}$ (Scheme 1).

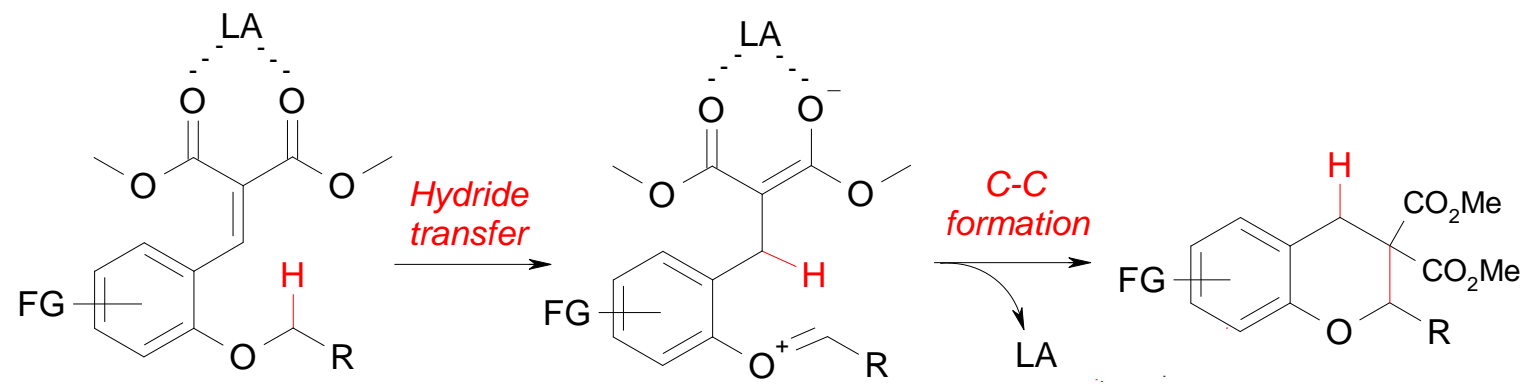

\footnotetext{
Scheme 1. The synthesis of 2-alkyl-3,3-dicarboxymethylchromanes from $O$-alkyl-substituted salicylic aldehydes.
}

The cyclization of such substrates proceeded upon moderate heating in the presence of Lewis acids, among which $\mathrm{Sc}(\mathrm{OTf})_{3}$ was found to be the most efficient one. ${ }^{9}$ It was suggested ${ }^{9,10}$ that the mechanism of these reactions included the stage of the hydride elimination from the OCH-moiety to produce a zwitter-ionic intermediate followed by its cyclization. With this respect, such reactions resemble cyclization of substituted tert-amines recently surveyed ${ }^{11}$ within the concept of the $t$-amino effect formulated by Meth-Cohn and Suschitsky. That type of processes with tertiary amines are often referred to as T-reactions in the literature ${ }^{8,12,13,14}$ (Scheme 2).

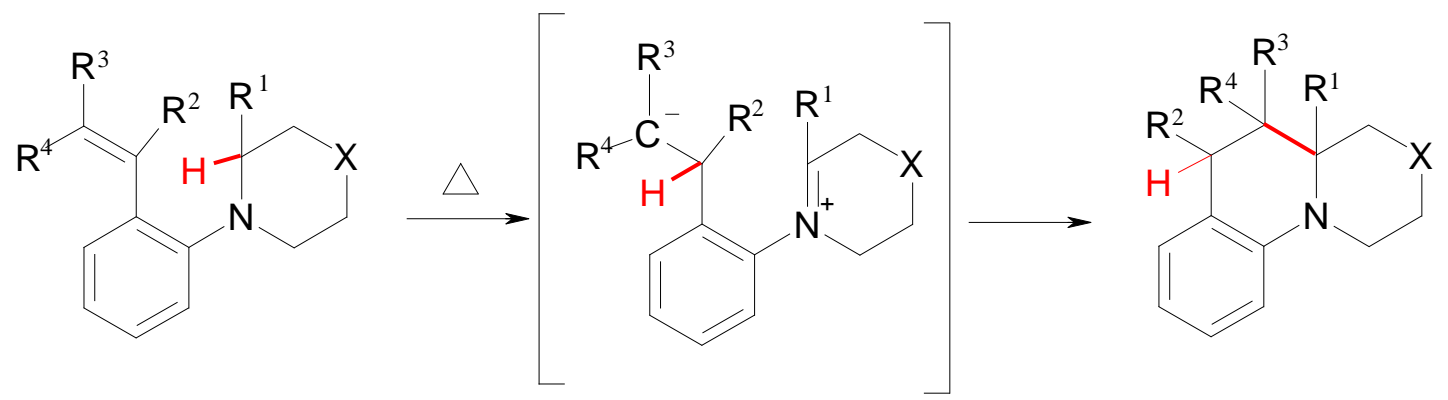


Scheme 2. The thermal isomerization of 2-vinyl- $N, N$-dialkylanilines into 1,2-annelated 1,2,3,4-tetrahydroquinolines.

Nevertheless, the specific cyclization depicted in Scheme 1 lies beyond the $t$-amino effect since the reaction substrates contain no alkylamino groups. On the other hand, these reactions can indeed be assigned to a common class due to the similarity of their mechanisms involving the hydride transfer from an aliphatic $\mathrm{CH}$-group to an unsaturated electron-deficient moiety.

\section{Results and discussion}

It is known that ortho-dialkylaminoarylidene derivatives of 1,3-dicarbonyl compounds, in particular, 5-arylidene barbituric acids (1), undergo a cyclization into the respective spirocyclic systems (2) ${ }^{8,15,16}$ (Scheme 3).
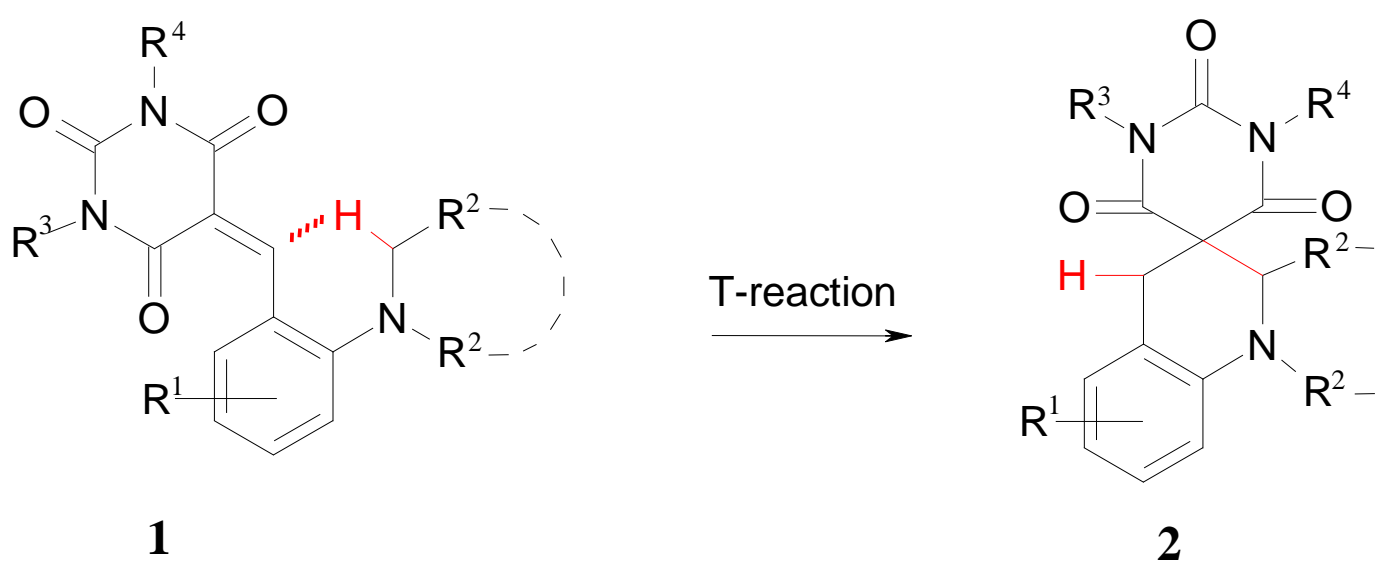

Scheme 3. The isomerization of 5-(2-dialkylaminoarylidene)barbituric acids into 5-spirobarbituric 1,2-annelated 1,2,3,4-tetrahydroquinolines.

The rate of the T-reaction for substrates similar to (1) is surprisingly high, the reaction proceeds much more readily than for derivatives of acyclic 1,3-dicarbonyl compounds. Indeed, the cyclization of reactants like (1) starts spontaneously at room temperature. Nevertheless, no cyclization of $O$ alkylated cyclic 1,3-dicarbonyl compounds has been reported so far, in contrast to their $N, N$ dialkylamino counterparts (1). In the present paper, we report on a newly discovered cyclization of 5-(2-benzyloxybenzylidene)barbituric acids (3) (Scheme 4).

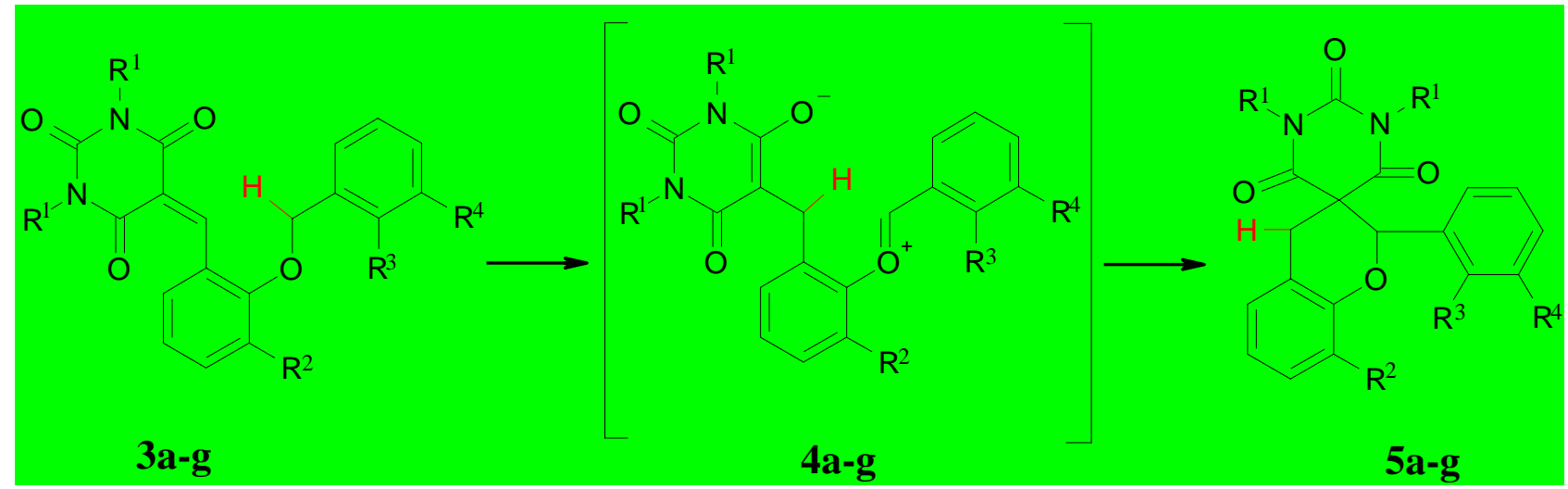

Scheme 4. The isomerization of 5-(2-benzyloxybenzylidene)barbituric acids 3a-g into spirocyclic chromane derivatives. $\mathrm{R}^{1}=\mathrm{R}^{2}=\mathrm{R}^{3}=\mathrm{R}^{4}=\mathrm{H}(\mathbf{a}) ; \mathrm{R}^{1}=\mathrm{Me}, \mathrm{R}^{2}=\mathrm{R}^{3}=\mathrm{R}^{4}=\mathrm{H}(\mathbf{b}) ; \mathrm{R}^{1}=\mathrm{R}^{3}=\mathrm{R}^{4}=\mathrm{H}$, $\mathrm{R}^{2}=\mathrm{OMe}(\mathbf{c}) ; \mathrm{R}^{1}=\mathrm{Me}, \mathrm{R}^{2}=\mathrm{OMe}, \mathrm{R}^{3}=\mathrm{R}^{4}=\mathrm{H}(\mathbf{d}) ; \mathrm{R}^{1}=\mathrm{R}^{2}=\mathrm{R}^{3}=\mathrm{H}, \mathrm{R}^{4}=\mathrm{Cl}(\mathbf{e})$; 


$$
\mathrm{R}^{1}=\mathrm{R}^{2}=\mathrm{R}^{4}=\mathrm{H}, \mathrm{R}^{3}=\mathrm{Cl}(\mathbf{f}) ; \mathrm{R}^{1}=\mathrm{R}^{2}=\mathrm{R}^{3}=\mathrm{H}, \mathrm{R}^{4}=\mathrm{OMe}(\mathbf{g})
$$

The starting substrates (3) were synthesized by the Knoevenagel condensation of barbituric acids with corresponding 2-alkoxybenzaldehydes (see Experimental section). We speculate that this cyclization follows a mechanism similar to the T-reaction shown in Scheme 4. Most probably, compounds 3 experience a hydride transfer giving rise to a zwitter-ionic intermediate (4) followed by its cyclization into the spirocyclic derivatives (5).

The molecular structure of one of the spirocyclic derivative synthesized via such a reaction is shown in Figure 1.

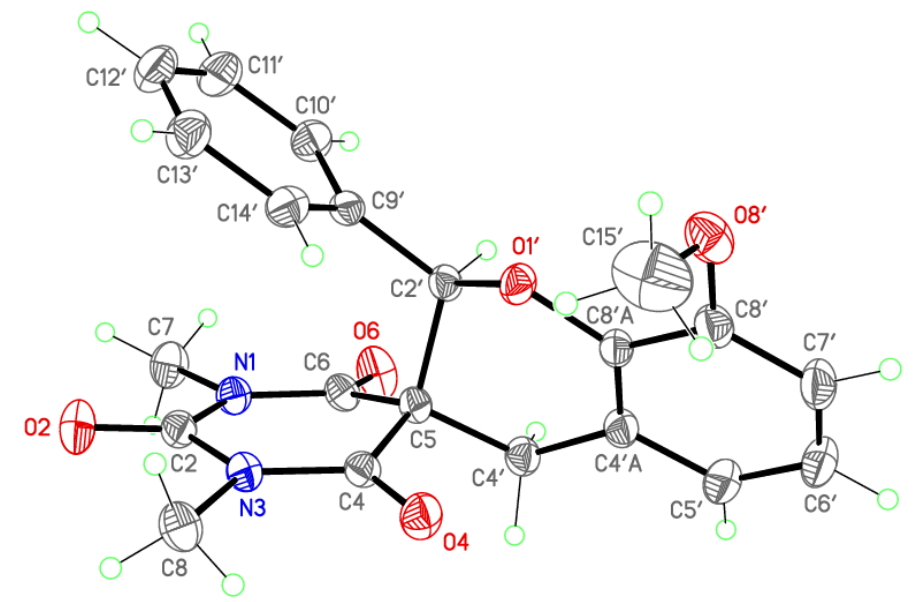

Figure 1. Molecular structure of $\mathbf{5 d}$.

The cyclization of 3a into the spirocyclic derivative 5a proceeded at $195{ }^{\circ} \mathrm{C}$ for $4 \mathrm{~h}$ in the solvent-free medium (Method A, see Experimental section). Similar conditions were required for the 1,3-dimethyl derivative $\mathbf{3 b}$. The reaction was facilitated by the presence of weak acids, it was possible to decrease the reaction temperature to $180{ }^{\circ} \mathrm{C}$ by adding succinic acid (Method $\mathrm{B}$, see Experimental section) (Table 1). Attempts to promote the reaction with Lewis acids $\left(\mathrm{BF}_{3}\right.$ or $\left.\mathrm{AlCl}_{3}\right)$ or strong protonic acid $\left(\mathrm{CF}_{3} \mathrm{COOH}, \mathrm{H}_{2} \mathrm{SO}_{4}, \mathrm{H}_{3} \mathrm{PO}_{4}\right)$ failed due to the apparent polymerization and resinification of the reaction mixture yielding no target product $\mathbf{5}$.

Table 1. Conditions of isomerization for substrates 3a-g, $\mathbf{6 c}$ and yields of respective spirocyclic products 5a-g, 7c

\begin{tabular}{|c|c|c|c|c|c|c|c|c|c|}
\hline \multirow[t]{2}{*}{ Reactant } & \multirow[t]{2}{*}{$\mathrm{R}^{1}$} & \multirow[t]{2}{*}{$\mathrm{R}^{2}$} & \multirow[t]{2}{*}{$\mathrm{R}^{3}$} & \multirow[t]{2}{*}{$\mathrm{R}^{4}$} & \multicolumn{2}{|c|}{ Reaction conditions } & \multirow[t]{2}{*}{ Product } & \multirow{2}{*}{$\begin{array}{l}\text { Method of } \\
\text { synthesis }\end{array}$} & \multirow{2}{*}{$\begin{array}{c}\text { Yield, } \\
\%\end{array}$} \\
\hline & & & & & $\mathrm{T},{ }^{\circ} \mathrm{C}$ & Time, $\mathrm{h}$ & & & \\
\hline $3 a$ & $\mathrm{H}$ & $\mathrm{H}$ & $\mathrm{H}$ & $\mathrm{H}$ & 195 & 4 & $5 \mathbf{a}$ & $\mathrm{A}^{\mathrm{a}}$ & 60 \\
\hline $\mathbf{3 b}$ & $\mathrm{Me}$ & $\mathrm{H}$ & $\mathrm{H}$ & $\mathrm{H}$ & 195 & 5 & $5 b$ & A & 45 \\
\hline 3b & $\mathrm{Me}$ & $\mathrm{H}$ & $\mathrm{H}$ & $\mathrm{H}$ & 180 & 4 & $5 b$ & $B^{b}$ & 80 \\
\hline $3 c$ & $\mathrm{H}$ & $\mathrm{OMe}$ & $\mathrm{H}$ & $\mathrm{H}$ & 180 & 2 & $5 c$ & A & 99 \\
\hline $3 c$ & $\mathrm{H}$ & $\mathrm{OMe}$ & $\mathrm{H}$ & $\mathrm{H}$ & 118 & 12 & $5 c$ & $C^{\mathbf{c}}$ & 97 \\
\hline 3d & $\mathrm{Me}$ & $\mathrm{OMe}$ & $\mathrm{H}$ & $\mathrm{H}$ & 118 & 40 & $5 d$ & $\mathrm{C}$ & 90 \\
\hline $3 e$ & $\mathrm{H}$ & $\mathrm{H}$ & $\mathrm{H}$ & $\mathrm{Cl}$ & 240 & 4 & $5 e$ & A & 84 \\
\hline $3 f$ & $\mathrm{H}$ & $\mathrm{H}$ & $\mathrm{Cl}$ & $\mathrm{H}$ & 240 & 4 & $5 f$ & $\mathrm{~A}$ & 35 \\
\hline $3 g$ & $\mathrm{H}$ & $\mathrm{H}$ & $\mathrm{H}$ & $\mathrm{OMe}$ & 190 & 4 & $5 g$ & $\mathrm{~A}$ & 61 \\
\hline $6 c$ & - & - & - & - & 180 & 2 & 7 & A & 95 \\
\hline $6 c$ & - & - & - & - & 118 & 48 & 7 & C & 80 \\
\hline
\end{tabular}


${ }^{a}$ Reaction by heating of solid substrate; ${ }^{b}$ Reaction by heating of solid substrate with admixture of succinic acid; ${ }^{c}$ Reaction in acetic acid solution under reflux (See Experimental section).

This means that the hydride elimination from the $\mathrm{OCH}_{2}$-group in compounds $\mathbf{3}$ as well as subsequent processes (Scheme 4) require harsher conditions as compared to the initiation of the T-reactions involving the alkylamino-group in reactants $\mathbf{1}$ (Scheme 3). Evidently, this is due to the lower basicity of the oxygen atom with respect to the amino nitrogen atom, which gives rise to a lower stability of the intermediate 4. Furthermore, X-ray crystallographic study revealed a distinct difference between the alkylamine intermediates $\mathbf{1}^{13}$ and their oxygen counterparts $\mathbf{3}$ (Figure 2 ).

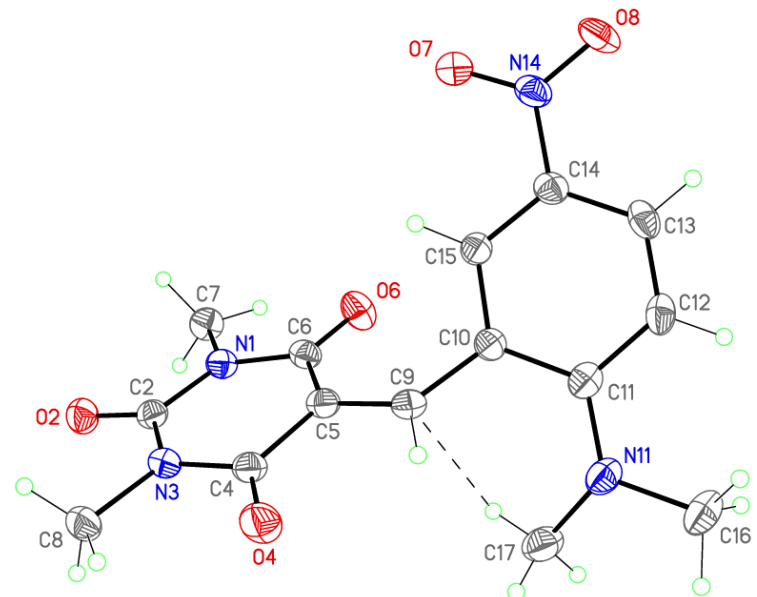

1a

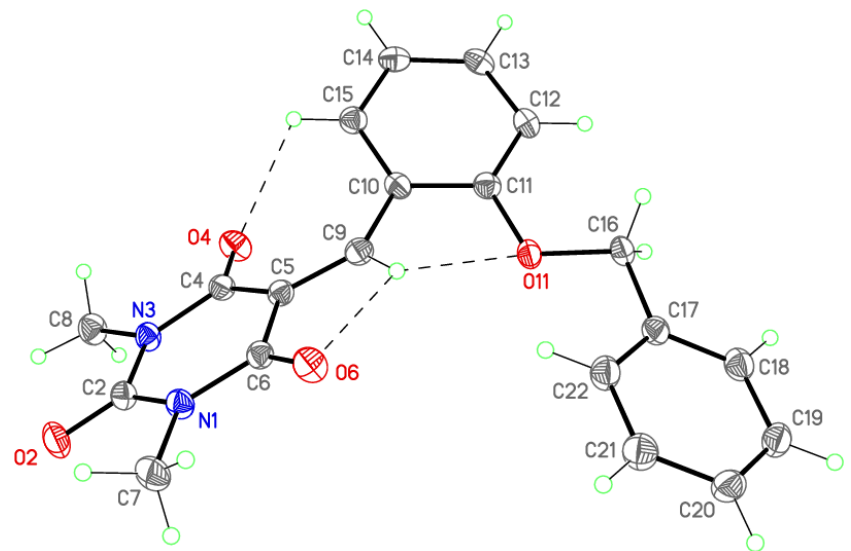

$3 \mathbf{b}$

Figure 2. X-Ray crystal structures of $\mathbf{1 a}$ and $\mathbf{3 b}$.

Substrates $\mathbf{1}$ are characterized by the presence of a close intramolecular C-H... $\pi$ contact between the vinyl carbon atom and the hydrogen atom prone to migration. ${ }^{13,16}$ Meanwhile, no such a contact is observed in substrates 3 .

5-(2-Benzyloxy-3-methoxy-5-benzylidene) barbituric acid derivatives $\mathbf{3 c}$ and $\mathbf{3 d}$ demonstrate a higher reactivity as compared to their analogs $\mathbf{3 a}$ and $\mathbf{3 b}$. The rearrangement of $\mathbf{3}$ into respective spiro-derivatives occurs at $118{ }^{\circ} \mathrm{C}$ upon boiling in acetic acid (Method C, see Experimental section) (Table 1). At first glance, this nearly 100-fold acceleration of the T-reaction can be assigned to the donor effect of the methoxy group in the aromatic ring, which stabilizes the zwitter-ionic intermediate 4. But X-ray crystallographic study of $\mathbf{3 d}$ revealed a very unusual molecular structure with the benzyloxy phenyl ring located nearly above the plane of the ylide double bond with the shortest intramolecular contact C9...C17 of 3.204(6) A (Figure 3). 


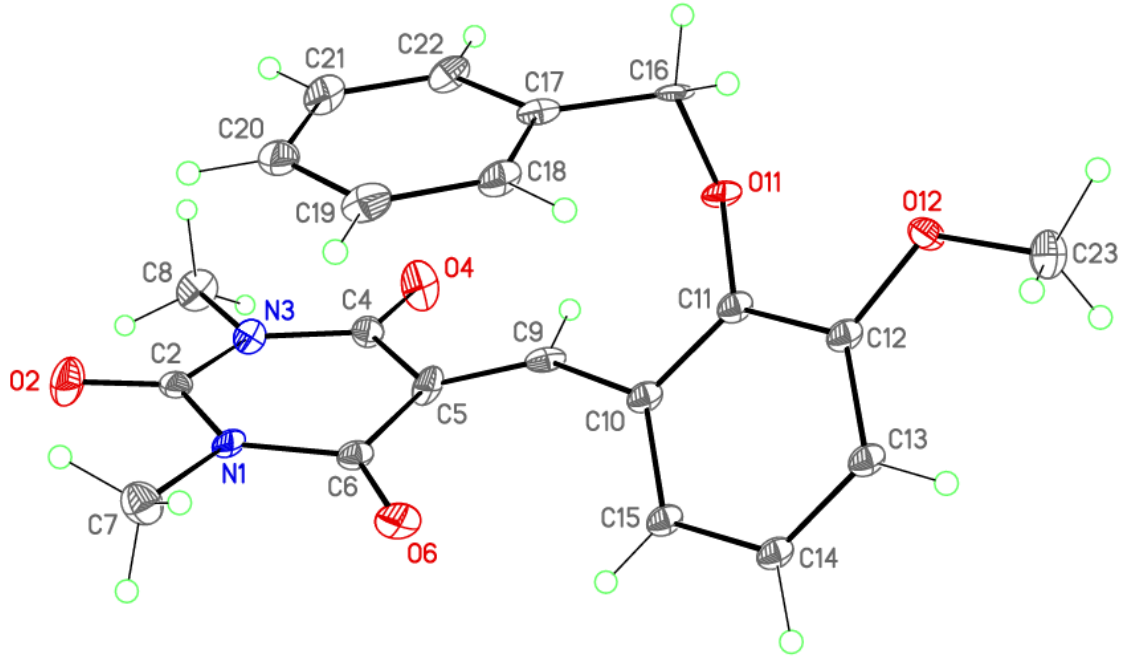

Figure 3. Molecular structure of 3d.

Such a geometry implies rather strong stacking interactions in 3d, which are expected to become even stronger for the zwitter-ionic intermediate $4 \mathbf{d}$ due to the localization of opposite charges on planes involved into the attractive interactions. This effect is presumably a main reason for the increased reactivity of substrates $\mathbf{3 c}, \mathbf{d}$.

An introduction of $\sigma$-acceptor functional groups into the phenyl ring of the benzyloxy group in reactants $\mathbf{3}$ gives rise to a pronounced reactivity inhibition. Indeed, the cyclization of 3-chloro- and 2-chloro derivatives $\mathbf{3 e}$ and $\mathbf{3 f}$ into the respective spiro-products $\mathbf{5 e}$ and $\mathbf{5 f}$ requires temperatures as high as $240^{\circ} \mathrm{C}$ (Table 1$)$.

The methoxybenzyl-substituted reactant $\mathbf{3 g}$ undergoes the cyclization into the respective spirocyclic product $\mathbf{5 g}$ nearly as facile as its unsubstituted analog $\mathbf{3 a}$.

The extension of substrate series to other 5-(2-alkoxybenzylidene)barbiturates showed that methoxy and ethoxy derivatives $6 \mathbf{a}$ and $\mathbf{6 b}$ do not rearrange even at $270{ }^{\circ} \mathrm{C}$. Meanwhile, 5-(2-isopropyloxybenzylidene) derivative $\mathbf{6 c}$ quite readily cyclizes into the respective spirocyclic product 7 (Scheme 5).

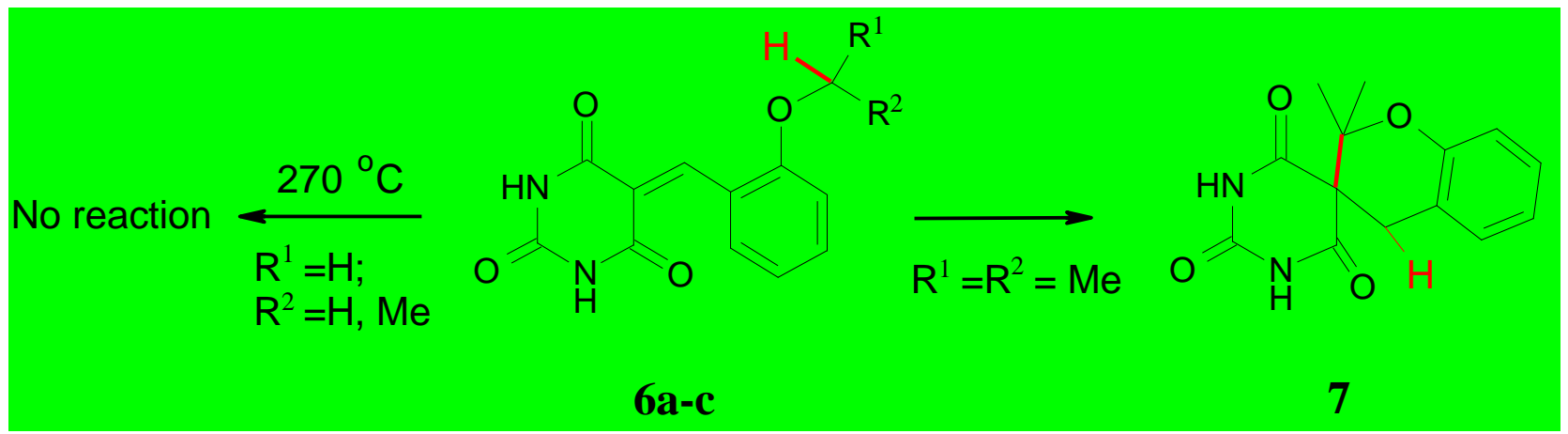

Scheme 5. The isomerization of 5-(2-alkoxybenzylidene)barbituric acids. $R^{1}=R^{2}=H(a) ; R^{1}=H$, $\mathrm{R}^{2}=\mathrm{Me}(\mathbf{b}) ; \mathrm{R}^{1}=\mathrm{R}^{2}=\mathrm{Me}(\mathbf{c})$; the yield of 7 is $95 \%$ by using of the Method $\mathrm{A}$ and $80 \%$ by the Method C (Table 1 and Experimental section)

The rearrangement of $\mathbf{6 c}$ into $\mathbf{7 c}$ can be realized either in the solvent-free medium or upon boiling in acetic acid (Table 1). This strongly suggests that a secondary alkoxy group is by far more reactive towards the T-reaction than a primary alkoxy group, which is probably due to a higher stability of the $\mathrm{O}^{+} \mathrm{CMe}_{2}$-cationic centre within the zwitter-ionic intermediate generated by the hydride transfer. 
In contrast to aforementioned 5-(2-benzyloxybenzylidene)barbiturates 3a-g (Scheme 4) and 6c (Scheme 5), the isomerization of 5-(2-allyloxybenzylidene)barbiturates 8a,b follows an alternative mechanism not involving a hydride transfer and affording cycloaddition products $\mathbf{9 a , b}$ (Scheme 6).

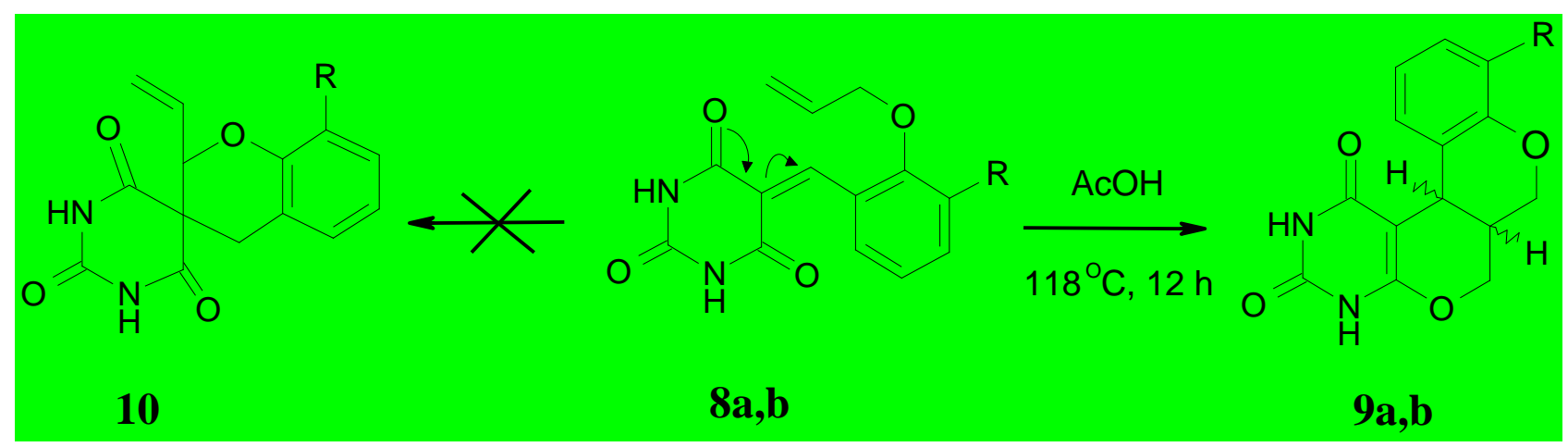

Scheme 6. The isomerization of 5-(2-allyloxybenzylidene)barbituric acids; $\mathrm{R}=\mathrm{H}(\mathbf{a}) ; \mathrm{R}=\mathrm{OMe}(\mathbf{b})$

The prevalence of the cycloaddition reaction pathway for derivatives $\mathbf{9 a}$ and $\mathbf{9 b}$ can be rationalized taking into account that similar cyclizations were reported for a number of 5-(2-allyloxybenzylidene)barbituriates. ${ }^{17}$

The Diels-Alder cycloaddition products 9 were isolated as a mixture of two isomers with the syn- and anti-configurations of protons at asymmetric carbon atoms with the former one being dominant (approximately $75 \%$ of $s y n$-isomer). No traces of anticipated spirocyclic products $\mathbf{1 0}$ were detected, which means that the cycloaddition reaction is much easier initiated than the hydride transfer. Interestingly, substrates $\mathbf{7}$ demonstrate totally different reactivity with this respect as compared to 2-allyloxy derivatives of malonic acid, which undergo cyclization to chromane derivatives via the hydride transfer (Scheme $1, \mathrm{R}=\mathrm{CH}=\mathrm{CH}_{2}$ ) as reported elsewhere. ${ }^{9}$

\section{Conclusion}

Therefore, the experimental evidence outlined above suggests that the reported cyclization of 5(2-alkoxybenzylidene)barbituric acids via a hydride transfer is indeed realized in the case of benzyloxy- or isopropyloxy-derived substrates. This finding unveils new facile routes to the synthesis of chromane-like spiro-pyrimidine derivatives that can be considered as analogs of flavonoids and related naturally occurring benzopyran systems.

\section{Experimental section}

\subsection{General}

The ${ }^{1} \mathrm{H}$ and ${ }^{13} \mathrm{C}$ NMR spectra were recorded on a AM-400 Bruker spectrometer (400 and $100 \mathrm{MHz}$, respectively). The purity of synthesized compounds was determined by elemental analysis and ${ }^{1} \mathrm{H}$ NMR spectroscopy.

The 5-(2-methoxybenzylidene)barbituric acid (6a) and 5-(2-ethoxybenzylidene)barbituric acid (6b) are commercially available. The 5-(2-isopropyloxybenzylidene)barbituric acid (6c) and 5-(2allyloxybenzylidene)barbituric acid (8a) are presented in catalog of ChemBrige Corporation. Other 5-(2-alkoxybenzylidene)barbituric acids 3a-g, 6c. 7a,b were synthesized from corresponding barbituric acids and O-alkyl derivatives of salicylic aldehyde (see p.p. 4.3). All of the used aldehydes are described in the literature, among them 2-benzyloxybenzaldehyde is commercially 
available, whereas other derivatives were synthesized by alkylation of salicylic aldehyde or $o$-vaniline (see p.p. 4.2). chloroform-methanol solutions.

X-ray structure determination. X-ray diffraction data were collected on the 'Belok' beamline of the Kurchatov Synchrotron Radiation Source (National Research Center "Kurchatov Institute", Moscow, Russian Federation) in the $\varphi$ scanning mode using a Rayonix SX165 CCD detector (for compounds 3b and 3d) and on a three-circle Bruker APEX-II CCD diffractometer using $\varphi$ and $\omega$ scanning mode (for compound 5d). The reflection intensities measured were corrected for absorption using the Scala program ${ }^{18}$ for compounds $\mathbf{3 b}$ and $\mathbf{3 d}$ and the $S A D A B S$ program ${ }^{19}$ for compound 5d. The data were indexed, integrated and scaled using the utility iMOSFLM in CCP4 program suite ${ }^{20}$ for compounds $\mathbf{3 b}$ and $\mathbf{3 d}$ and Bruker APEX2 program $^{21}$ for compound $\mathbf{5 d}$. For details, see Supporting Information. The structures were determined by direct methods and refined by full-matrix least squares technique on $F^{2}$ with anisotropic displacement parameters for nonhydrogen atoms. The hydrogen atoms were placed in calculated positions and refined within the riding model with fixed isotropic displacement parameters $\left[U_{\text {iso }}(\mathrm{H})=1.5 U_{\text {eq }}(\mathrm{C})\right.$ for the methyl groups and $1.2 U_{\mathrm{eq}}(\mathrm{C})$ for the other groups]. The calculations were carried out using the SHELXTL program ${ }^{22}$.

Crystallographic data for the investigated compounds have been deposited with the Cambridge Crystallographic Data Center, CCDC 1502465 (3b), CCDC 1502466 (3d), and CCDC 1502467 (5d). Copies of this information may be obtained free of charge from the Director, CCDC, 12 Union Road, Cambridge CB2 1EZ, UK (fax: +44 1223 336033; e-mail: deposit@ccdc.cam.ac.uk or www.ccdc.cam.ac.uk).

\subsection{General procedure for synthesis of 2-alkoxy benzaldehydes.}

To the stirred solution of salicylic aldehyde $(0.1 \mathrm{~mol})$ (or 2-hydroxy-3-methoxybenzaldehyde (o-vanilin)) in DMF $(40 \mathrm{~mL})$, dry $\mathrm{K}_{2} \mathrm{CO}_{3}(0.12 \mathrm{~mol})$ was added and the mixture was stirred for $15 \mathrm{~min}$ at $20^{\circ} \mathrm{C}$. Then corresponding alkyl halide $(0.1 \mathrm{~mol})$ was added, and the reaction mixture was stirred for $12 \mathrm{~h}$ at $60{ }^{\circ} \mathrm{C}$. The cooled reaction mixture was poured into water $(250 \mathrm{~mL})$ and extracted by $\mathrm{CH}_{2} \mathrm{Cl}_{2}(40 \mathrm{~mL})$. An organic layer was separated, washed with $\mathrm{NH}_{4} \mathrm{OH} 5 \%(5 \times 25 \mathrm{~mL})$ and water $(50 \mathrm{~mL})$, and dried over $\mathrm{Na}_{2} \mathrm{SO}_{4}$. A solvent was removed in vacuo and the residue was crystallized by adding of cyclohexane (in case of the solid compounds, see p.p. 4.2.1-4.2.4), or distilled in vacuo $1 \mathrm{~mm}$. $\mathrm{Hg}$ (in case of liquids, see p.p. 4.2.5-4.2.7).

4.2.1. 2-Benzyloxy-3-methoxybenzaldehyde (Acros Organics, cat. № 42567). It was prepared from from o-vanilin and benzyl chloride in $77 \%$ yield. Colorless crystals, m.p. $48^{\circ} \mathrm{C}$.

4.2.2. 2-(2-Chlorobenzyloxy)benzaldehyde (AK Scientific, cat. № 5081AE). It was prepared from salicylic aldehyde and 2-chlorobenzyl chloride in $82 \%$ yield. Colorless crystals, m.p. $88{ }^{\circ} \mathrm{C}$.

4.2.3. 2-(3-Chlorobenzyloxy)benzaldehyde (AK Scientific, cat. № 2696AE). It was prepared from salicylic aldehyde and 3-chlorobenzyl chloride in $79 \%$ yield. Colorless crystals, m.p. $42{ }^{\circ} \mathrm{C}$.

4.2.4. 2-(3-Methoxybenzyloxy)benzaldehyde (ChemBrige Co cat. № 3014101). It was prepared from salicylic aldehyde and 2-chlorobenzyl chloride in $85 \%$ yield. Colorless crystals, m.p. $73{ }^{\circ} \mathrm{C}$.

4.2.5. 2-Isopropyloxybenzaldehyde (AK Scientific, cat. № 7011AD). It was prepared from salicylic aldehyde and 2-iodopropane in 66\% yield. Colorless oil.

4.2.6. 2-Allyloxybenzaldehyde (Acros Organics, cat. № 38083). It was prepared from salicylic aldehyde and allyl bromide in $87 \%$ yield. Colorless oil.

\subsubsection{2-Allyloxy-3-methoxybenzaldehyd (AK Scientific, cat. № 7908AD).. It was prepared from o-}


vanilin and allyl bromide in $83 \%$ yield. Colorless oil.

\subsection{General procedure for synthesis of 2-alkoxybenzylidene barbiturates 3a-g, $6 \mathrm{c}, 7 \mathrm{a}, \mathrm{b}$.}

Barbituric acid (10 mmol) (or 1,3-dimethylbarbituric acid) were dissolved in water $(5 \mathrm{~mL})$ under heating, and the solution of corresponding aldehyde $(10 \mathrm{mmol})$ in ethanol $(10 \mathrm{~mL})$ of was added. The reaction mixture was stirred for $5 \mathrm{~min}$ at $75{ }^{\circ} \mathrm{C}$ and allowed to stay at room temperature for $2 \mathrm{~h}$. A precipitate was filtered out, washed with $50 \%$ ethanol and dried in air.

\subsubsection{5-(2-Benzyloxybenzylidene)barbituric acid (3a).}

It was prepared from barbituric acid and 2-benzyloxybenzaldehyde in $96 \%$ yield. Yellow crystals, m.p. 204-206 ${ }^{\circ} \mathrm{C}$; [Found: $\mathrm{C}, 66.87 ; \mathrm{H}, 4.40 ; \mathrm{N}, 8.61 . \mathrm{C}_{18} \mathrm{H}_{14} \mathrm{~N}_{2} \mathrm{O}_{4}$ requires $\mathrm{C}, 67.08 ; \mathrm{H}, 4.38 ; \mathrm{N}$, $8.69 \%] ; v_{\max }(\mathrm{KBr}) 3062,2856,1756,1664,1562,1393,1294,1230,1020,841,789,753,728$, $659,506 \mathrm{~cm}^{-1} ; \delta_{\mathrm{H}}\left(400 \mathrm{MHz}, \mathrm{DMSO}-d_{6}\right) 11.35(1 \mathrm{H}, \mathrm{s}, \mathrm{NH}), 11.18(1 \mathrm{H}, \mathrm{s}, \mathrm{N} \underline{\mathrm{H}}), 8.58(1 \mathrm{H}, \mathrm{s}$, $\mathrm{C} \underline{\mathrm{H}}=\mathrm{C}), 8.01(1 \mathrm{H}, \mathrm{d}, J 8.2 \mathrm{~Hz}, \operatorname{Ar} \underline{\mathrm{H}}), 7.29-7.53(6 \mathrm{H}, \mathrm{m}, \operatorname{Ar} \underline{\mathrm{H}}), 7.19(1 \mathrm{H}, \mathrm{d}, J 8.2 \mathrm{~Hz}, \operatorname{Ar} \underline{\mathrm{H}}), 7.00$ $(1 \mathrm{H}, \mathrm{t}, J 8.2 \mathrm{~Hz}, \operatorname{Ar} \underline{\mathrm{H}}), 5.22\left(2 \mathrm{H}, \mathrm{s}, \mathrm{OC} \underline{\mathrm{H}}_{2}\right) ; \delta_{\mathrm{C}}\left(100 \mathrm{MHz}, \mathrm{DMSO}-d_{6}\right) 163.8,181.9,158.6,150.7$, $150.5,137.1,134.4,133.0,129.0,128.4,127.9,122.5,120.2,119.3,112.9,70.4$.

\subsubsection{1,3-Dimethyl-5-(2-benzyloxybenzylidene)barbituric acid (3b).}

It was prepared from 1,3-dimethylbarbituric acid and 2-benzyloxybenzaldehyde in $95 \%$ yield. Yellow crystals, m.p. $160-161{ }^{\circ} \mathrm{C}$. [Found: $\mathrm{C}, 68.41 ; \mathrm{H}, 5.22 ; \mathrm{N}, 7.93 . \mathrm{C}_{20} \mathrm{H}_{18} \mathrm{~N}_{2} \mathrm{O}_{4}$ requires $\mathrm{C}$, 68.56; H, 5.18; N, 8.00\%]; $v_{\max }(\mathrm{KBr}) 3676,3062,3031,1730,1667,1587,1453,1373,1253$, $1160,1080,789,761,692,593,472 \mathrm{~cm}^{-1} ; \delta_{\mathrm{H}}\left(400 \mathrm{MHz}, \mathrm{CDCl}_{3}\right) 8.97(1 \mathrm{H}, \mathrm{s}, \mathrm{C} \underline{\mathrm{H}}=\mathrm{C}), 8.03(1 \mathrm{H}, \mathrm{d}$, $J 8.2 \mathrm{~Hz}, \operatorname{Ar} \underline{\mathrm{H}}), 7.30-7.51$ (6 H, m, Ar$\underline{\mathrm{H}}), 7.03$ (2 H, m, $\operatorname{Ar} \underline{\mathrm{H}}), 5.20$ (2 H, s, OC$\left.\underline{\mathrm{H}}_{2}\right), 3.41(3 \mathrm{H}, \mathrm{s}$, $\mathrm{NMe}), 3.24$ (3 H, s, NMe); $\delta_{\mathrm{C}}\left(100 \mathrm{MHz}, \mathrm{CDCl}_{3}\right)$ 162.1, 160.0, 156.8, 149.9, 149.5, 136.0, 133.3, $130.9,128.1,127.4,127.0,122.1,119.7,118.8,112.5,71.1,28.8,28.1$.

\subsubsection{5-(2-Benzyloxy-3-methoxybenzylidene)barbituric acid (3c).}

It was prepared from barbituric acid and 2-benzyloxy-3-methoxybenzaldehyde in $95 \%$ yield. Yellow crystals, m.p. $203-204{ }^{\circ} \mathrm{C}$. [Found: C, 64.69; H, 4.60; N, 7.89. $\mathrm{C}_{19} \mathrm{H}_{16} \mathrm{~N}_{2} \mathrm{O}_{5}$ requires C, 64.77; $\mathrm{H}$, 4.58 ; N, 7.95\%]; $v_{\max }(\mathrm{KBr}) 3059,2841,1752,1662,1548,1395,1264,1230,1081,958,914,836$, $751,699,507 \mathrm{~cm}^{-1} ; \delta_{\mathrm{H}}\left(400 \mathrm{MHz}, \mathrm{DMSO}-d_{6}\right) 11.32(1 \mathrm{H}, \mathrm{s}, \mathrm{N} \underline{\mathrm{H}}), 11.12(1 \mathrm{H}, \mathrm{s}, \mathrm{NH}), 8.34(1 \mathrm{H}, \mathrm{s}$, $\mathrm{C} \underline{\mathrm{H}}=\mathrm{C}), 7.50(1 \mathrm{H}, \mathrm{d}, J 8.1 \mathrm{~Hz}, \operatorname{Ar} \underline{\mathrm{H}}), 7.32(5 \mathrm{H}, \mathrm{m}, \operatorname{Ar} \underline{\mathrm{H}}), 7.24(1 \mathrm{H}, \mathrm{d}, J 8.1 \mathrm{~Hz}, \operatorname{Ar} \underline{\mathrm{H}}), 7.10(1 \mathrm{H}, \mathrm{t}$, $J 8.1 \mathrm{~Hz}, \operatorname{Ar} \underline{\mathrm{H}}), 5.01\left(2 \mathrm{H}, \mathrm{s}, \mathrm{OC} \underline{\underline{H}}_{2}\right), 3.89(3 \mathrm{H}, \mathrm{s}, \mathrm{OMe}) ; \delta_{\mathrm{C}}\left(100 \mathrm{MHz}, \mathrm{DMSO}-d_{6}\right)$ 163.6, 161.5, $152.5,150.9,150.7,147.6,137.2,129.2,128.7,128.5,128.3,123.9,123.6,120.1,116.7,75.4,56.5$.

4.3.4. 1,3-Dimethyl-5-(2-benzyloxy-3-methoxy-benzylidene)barbituric acid (3d).

It was prepared from 1,3-dimethylbarbituric acid and 2-benzyloxy-3-methoxybenzaldehyde in 95\% yield. Colorless crystals, m.p. $160-161^{\circ} \mathrm{C}$. [Found: C, 66.15; H, 5.33; N, 7.29. $\mathrm{C}_{21} \mathrm{H}_{20} \mathrm{~N}_{2} \mathrm{O}_{5}$ requires $\mathrm{C}, 66.31 ; \mathrm{H}, 5.30 ; \mathrm{N}, 7.36 \%$ ]; $v_{\max }(\mathrm{KBr}) 3006,2955,1736,1667,1574,1476,1379,1277,1184$, $1107,788,749,703,610,470 \mathrm{~cm}^{-1} ; \delta_{\mathrm{H}}\left(400 \mathrm{MHz}, \mathrm{CDCl}_{3}\right) 8.97(1 \mathrm{H}, \mathrm{s}, \mathrm{C} \underline{\mathrm{H}}=\mathrm{C}), 7.40(1 \mathrm{H}, \mathrm{d}, J 8.1$ $\mathrm{Hz}, \operatorname{Ar} \underline{\mathrm{H}}), 7.28(5 \mathrm{H}, \mathrm{m}, \operatorname{Ar} \underline{\mathrm{H}}), 7.12(2 \mathrm{H}, \mathrm{m}, \operatorname{Ar} \underline{\mathrm{H}}), 5.07$ (2 H, s, OC $\left.\underline{\mathrm{H}}_{2}\right), 3.95$ (3 H, s, OMe $), 3.40$ (3 H, s, NMe), 3.27 (3 H, s, NMe); $\delta_{\mathrm{C}}\left(100 \mathrm{MHz}, \mathrm{CDCl}_{3}\right) 161.9,159.8,155.7,152.4,151.3,147.4$, $136.8,129.3,128.8,128.2,128.0,123.4,123.3,118.3,115.9,76.0,56.0,28.8,28.2$.

\subsubsection{5-(2-(3-Chlorobenzyloxy)benzylidene)barbituric acid (3e).}

It was prepared from barbituric acid and 2-(3-chlorobenzyloxy)benzaldehyde in $97 \%$ yield. Yellow crystals, m.p. $240-241{ }^{\circ} \mathrm{C}$. [Found: $\mathrm{C}$, 60.49; $\mathrm{H}, 3.74 ; \mathrm{N} 7.80 . \mathrm{C}_{18} \mathrm{H}_{13}{ }^{35} \mathrm{ClN}_{2} \mathrm{O}_{4}$ requires $\mathrm{C}, 60.60$; $\mathrm{H}, 3.67 ; \mathrm{N}, 7.85 \%$; $v_{\max }(\mathrm{KBr}) 3195,3066,1759,1683,1582,1486,1431,1381,1297,1243,1122$, 935, 828, 746, 704, 551, $505 \mathrm{~cm}^{-1} ; \delta_{\mathrm{H}}\left(400 \mathrm{MHz}, \mathrm{DMSO}-d_{6}\right) 11.36(1 \mathrm{H}, \mathrm{s}, \mathrm{N} \underline{\mathrm{H}}), 11.17(1 \mathrm{H}, \mathrm{s}$, $\mathrm{N} \underline{\underline{H}}), 8.59(1 \mathrm{H}, \mathrm{s}, \mathrm{C} \underline{\mathrm{H}}=\mathrm{C}), 8.01(1 \mathrm{H}, \mathrm{d}, J 8.2 \mathrm{~Hz}, \operatorname{ArH}), 7.48(5 \mathrm{H}, \mathrm{m}, \operatorname{Ar} \underline{\mathrm{H}}), 7.21(1 \mathrm{H}, \mathrm{d}, J 8.2 \mathrm{~Hz}$, $\operatorname{Ar} \underline{\mathrm{H}}), 7.01(1 \mathrm{H}, \mathrm{t}, J \mathbf{J} .2 \mathrm{~Hz}, \operatorname{\mathrm {Ar}} \underline{\mathrm{H}}), 5.25\left(2 \mathrm{H}, \mathrm{s}, \mathrm{OC}_{\underline{\mathrm{H}}}\right) ; \delta_{\mathrm{C}}\left(100 \mathrm{MHz}, \mathrm{DMSO}-d_{6}\right) 163.8,161.9$, 
$158.3,150.7,150.2,139.7,134.3,133.7,133.0,130.9,128.3,127.6,126.4,122.5,120.5,119.4$, $112.9,69.5$.

4.3.6. 5-(2-(2-Chlorobenzyloxy)benzylidene)barbituric acid (3f).

It was prepared from barbituric acid and 2-(2-chlorobenzyloxy)benzaldehyde in $97 \%$ yield. Yellow crystals, m.p. $239-240{ }^{\circ} \mathrm{C}$. [Found: $\mathrm{C}, 60.64 ; \mathrm{H}, 3.70 ; \mathrm{N}, 7.79 . \mathrm{C}_{18} \mathrm{H}_{13}{ }^{35} \mathrm{ClN}_{2} \mathrm{O}_{4}$ requires $\mathrm{C}, 60.60$; $\mathrm{H}, 3.67 ; \mathrm{N}, 7.85 \%$; $v_{\max }(\mathrm{KBr}) 3202,3079,1760,1681,1592,1486,1431,1382,1298,1253,1121$, $1035,935,788,744,540,505 \mathrm{~cm}^{-1} ; \delta_{\mathrm{H}}\left(400 \mathrm{MHz}, \mathrm{DMSO}-d_{6}\right) 11.33(1 \mathrm{H}, \mathrm{s}, \mathrm{N} \underline{\mathrm{H}}), 11.17(1 \mathrm{H}, \mathrm{s}$, $\mathrm{N} \underline{\mathrm{H}}), 8.58(1 \mathrm{H}, \mathrm{s}, \mathrm{C} \underline{\mathrm{H}}=\mathrm{C}), 8.00(1 \mathrm{H}, \mathrm{d}, J 8.2 \mathrm{~Hz}, \operatorname{Ar} \underline{\mathrm{H}}), 7.54(2 \mathrm{H}, \mathrm{m}, \hat{\operatorname{Ar}} \underline{\mathrm{H}}), 7.40(2 \mathrm{H}, \mathrm{m}, \operatorname{Ar} \underline{\mathrm{H}})$, $7.22(1 \mathrm{H}, \mathrm{d}, J 8.2 \mathrm{~Hz}, \operatorname{Ar} \underline{\mathrm{H}}), 7.02(1 \mathrm{H}, \mathrm{t}, J 8.2 \mathrm{~Hz}, \operatorname{Ar} \underline{\mathrm{H}}), 5.29\left(2 \mathrm{H}, \mathrm{s}, \mathrm{OC} \underline{\underline{H}}_{2}\right) ; \delta_{\mathrm{C}}(100 \mathrm{MHz}$, DMSO- $\left.d_{6}\right) 163.8,161.9,158.2,150.7,150.4,134.4,134.3,133.1,133.0,130.5(2 C), 130.0,127.9$, 122.7, 120.5, 119.4, 112.9, 68.2.

\subsubsection{5-(2-(3-Methoxybenzyloxy)benzylidene)barbituric acid (3g).}

It was prepared from barbituric acid and 2-(3-methoxybenzyloxy)benzaldehyde in $94 \%$ yield. Yellow crystals, m.p. $174-175{ }^{\circ} \mathrm{C}$. [Found: $\mathrm{C}$, 64.65; $\mathrm{H}, 4.61 ; \mathrm{N}, 7.87 . \mathrm{C}_{19} \mathrm{H}_{16} \mathrm{~N}_{2} \mathrm{O}_{5}$ requires $\mathrm{C}$, 64.77; H, 4.58; N 7.95\%]; $v_{\max }(\mathrm{KBr}) 3223,1746,1676,1600,1452,1384,1248,1031,932,783$, $758,692,503 \mathrm{~cm}^{-1} ; \delta_{\mathrm{H}}\left(400 \mathrm{MHz}\right.$, DMSO- $\left.d_{6}\right) 11.37(1 \mathrm{H}, \mathrm{s}, \mathrm{N} \underline{\underline{H}}), 11.17(1 \mathrm{H}, \mathrm{s}, \mathrm{N} \underline{\underline{H}}), 8.61(1 \mathrm{H}, \mathrm{s}$, $\mathrm{C} \underline{\mathrm{H}}=\mathrm{C}), 8.02(1 \mathrm{H}, \mathrm{d}, J 8.2 \mathrm{~Hz}, \operatorname{Ar} \underline{\mathrm{H}}), 7.50(1 \mathrm{H}, \mathrm{t}, J 8.2 \mathrm{~Hz}, \operatorname{Ar} \underline{\mathrm{H}}), 7.31(1 \mathrm{H}, \mathrm{t}, J 8.2 \mathrm{~Hz}, \operatorname{Ar} \underline{\mathrm{H}})$, $7.20(1 \mathrm{H}, \mathrm{d}, J 8.2 \mathrm{~Hz}, \operatorname{Ar} \underline{\mathrm{H}}), 7.01(3 \mathrm{H}, \mathrm{m}, \operatorname{Ar} \underline{\mathrm{H}}), 6.90(1 \mathrm{H}, \mathrm{dd}, J$ 8.2, $2.4 \mathrm{~Hz}, \operatorname{Ar} \underline{\mathrm{H}}), 5.27(2 \mathrm{H}, \mathrm{s}$, $\left.\mathrm{OCH}_{2}\right), 3.78$ (3H, s, OMe); $\delta_{\mathrm{C}}\left(100 \mathrm{MHz}, \mathrm{DMSO}-d_{6}\right) 163.9,161.9,159.9,158.5,150.7,150.4$, 138.7, 134.4, 133.0, 130.1, 122.5, 120.3, 119.9, 119.3, 114.1, 113.1, 112.9, 70.3, 55.5.

4.3.8. 5-(2-Isopropyloxybenzylidene)barbituric acid (6c) (ChemBrige Co cat. № 5316075).

It was prepared from barbituric acid and 2-isopropyloxybenzaldehyde in $82 \%$ yield. Yellow crystals, m.p. 218-219 ${ }^{\circ} \mathrm{C}$. [Found: $\mathrm{C}, 61.29 ; \mathrm{H}, 5.15 ; \mathrm{N}, 10.19 . \mathrm{C}_{14} \mathrm{H}_{14} \mathrm{~N}_{2} \mathrm{O}_{4}$ requires $\mathrm{C}, 61.31 ; \mathrm{H}, 5.14 ; \mathrm{N}$, $10.21 \%] ; \delta_{\mathrm{H}}\left(400 \mathrm{MHz}\right.$, DMSO- $\left.d_{6}\right) 11.32(1 \mathrm{H}, \mathrm{s}, \mathrm{NH}), 11.14(1 \mathrm{H}, \mathrm{s}, \mathrm{NH}), 8.51(1 \mathrm{H}, \mathrm{s}, \mathrm{C} \underline{\mathrm{H}}=\mathrm{C})$, $8.00(1 \mathrm{H}, \mathrm{dd}, J$ J 8.2, $2.2 \mathrm{~Hz}, \operatorname{Ar} \underline{\mathrm{H}}), 7.49(1 \mathrm{H}, \mathrm{dd}, J 8.2,2.2 \mathrm{~Hz}, \operatorname{Ar} \underline{\mathrm{H}}), 7.13(1 \mathrm{H}, \mathrm{d}, J 8.0 \mathrm{~Hz}, \operatorname{Ar} \underline{\mathrm{H}})$, $6.95(1 \mathrm{H}, \mathrm{t}, J 8.0 \mathrm{~Hz}, \mathrm{Ar} \underline{\mathrm{H}}), 4.73\left(1 \mathrm{H}, \mathrm{m}, \mathrm{C} \underline{\underline{H} \mathrm{He}_{2}}\right), 1.30\left(6 \mathrm{H}, \mathrm{d}, J 5.9 \mathrm{~Hz}, \mathrm{CHMe}_{2}\right)$.

\subsubsection{5-(2-Allyloxybenzylidene)barbituric acid (8a) (ChemBrige Co cat. № 5567165).}

It was prepared from barbituric acid and 2-allyloxybenzaldehyde in $92 \%$ yield. Yellow crystals, m.p. 195-196 ${ }^{\circ} \mathrm{C}$. [Found: $\mathrm{C}, 61.74 ; \mathrm{H}, 4.47 ; \mathrm{N}, 10.25 . \mathrm{C}_{14} \mathrm{H}_{12} \mathrm{~N}_{2} \mathrm{O}_{4}$ requires $\mathrm{C}$, 61.76; H, 4.44; $\mathrm{N}, 10.29 \%] ; \delta_{\mathrm{H}}\left(400 \mathrm{MHz}\right.$, DMSO-$\left.d_{6}\right) 11.34(1 \mathrm{H}, \mathrm{s}, \mathrm{N} \underline{\mathrm{H}}) .11 .16(1 \mathrm{H}, \mathrm{s}, \mathrm{N} \underline{\underline{H}}), 8.56(1 \mathrm{H}, \mathrm{s}, \mathrm{C} \underline{\mathrm{H}}=\mathrm{C})$, $8.01(1 \mathrm{H}, \mathrm{dd}, J$ 8.2, $2.1 \mathrm{~Hz}, \mathrm{Ar} \underline{\mathrm{H}}), 7.50(1 \mathrm{H}, \mathrm{t}, J 8.2 \mathrm{~Hz}, \operatorname{Ar} \underline{\mathrm{H}}), 7.11$ (1 H, d, $J 8.2 \mathrm{~Hz}, \mathrm{Ar} \underline{\mathrm{H}}), 6.99$ (1 $\mathrm{H}, \mathrm{t}, J 8.2 \mathrm{~Hz}, \mathrm{Ar} \underline{\mathrm{H}}), 6.07\left(1 \mathrm{H}, \mathrm{m}, \mathrm{CH}=\mathrm{CH}_{2}\right), 5.43(1 \mathrm{H}, \mathrm{dd}, J 17.1,1.9 \mathrm{~Hz}, \mathrm{CH}=\mathrm{CH} \underline{\mathrm{H}}), 5.30(1 \mathrm{H}$, dd, $J 10.5,1.8 \mathrm{~Hz}, \mathrm{CH}=\mathrm{C} \underline{\mathrm{HH}}), 4.70\left(2 \mathrm{H}, \mathrm{d}, J 5.1 \mathrm{~Hz}, \mathrm{OC}_{2}\right)$.

\subsubsection{5-(2-Allyloxy-3-methoxybenzylidene)barbituric acid (8b).}

It was prepared from barbituric acid and 2-allyloxy-3-methoxybenzaldehyde in $90 \%$ yield. Yellow crystals, m.p. $200-201{ }^{\circ} \mathrm{C}$. [Found: $\mathrm{C}, 59.56 ; \mathrm{H}, 4.70 ; \mathrm{N}, 9.21 . \mathrm{C}_{15} \mathrm{H}_{14} \mathrm{~N}_{2} \mathrm{O}_{5}$ requires $\mathrm{C}, 59.60 ; \mathrm{H}$, 4.67; N, 9.27\%]; $v_{\max }(\mathrm{KBr}) 3199,3071,2844,1743,1676,1558,1444,1391,1270,1223,1077$, 976, 858, 794, 695, $500 \mathrm{~cm}^{-1} ; \delta_{\mathrm{H}}\left(400 \mathrm{MHz}\right.$, DMSO-d $\left.d_{6}\right) 11.36(1 \mathrm{H}, \mathrm{s}, \mathrm{N} \underline{\mathrm{H}}), 11.17(1 \mathrm{H}, \mathrm{s}, \mathrm{N} \underline{\mathrm{H}})$, $8.46(1 \mathrm{H}, \mathrm{s}, \mathrm{C} \underline{\mathrm{H}}=\mathrm{C}), 7.51(1 \mathrm{H}, \mathrm{d}, J 8.2 \mathrm{~Hz}, \operatorname{Ar} \underline{\mathrm{H}}), 7.08(1 \mathrm{H}, \mathrm{t}, J 8.2 \mathrm{~Hz}, \operatorname{Ar} \underline{\mathrm{H}}), 7.21(1 \mathrm{H}, \mathrm{d}, J 8.2$ $\mathrm{Hz}, \mathrm{ArH}), 5.99\left(1 \mathrm{H}, \mathrm{m}, \mathrm{CH}=\mathrm{CH}_{2}\right), 5.33(1 \mathrm{H}, \mathrm{dd}, J 16.1,2.0 \mathrm{~Hz}, \mathrm{CH}=\mathrm{CHH}), 5.20(1 \mathrm{H}, \mathrm{dd}, J 10.6$, $1.8 \mathrm{~Hz}, \mathrm{CH}=\mathrm{CH} \underline{\mathrm{H}}), 4.53\left(2 \mathrm{H}, \mathrm{d}, J 5.5 \mathrm{~Hz}, \mathrm{OCH}_{2}\right), 3.84(3 \mathrm{H}, \mathrm{s}, \mathrm{OMe}) ; \delta_{\mathrm{C}}\left(100 \mathrm{MHz}, \mathrm{DMSO}-d_{6}\right)$ 163.6, 161.7, 152.3, 150.8, 150.7, 147.9, 134.4, 128.0, 123.8, 123.4, 120.4, 118.4, 116.7, 74.5, 56.4.

\subsection{General Method A - the procedure for synthesis of 5-spiro-3'-cromans 5a-q and 7 (Table 1) without solvent}


The derivative of 5-benzylidenebarbituric acid (3a-g, 6c) $(2 \mathrm{mmol})$ of was heated in sealed ampoule under inert atmosphere. After cooling, the solid product was crushed and washed by hot conditions of reaction (the temperature and time of heating) and yields see in Table 1.

\subsubsection{2,4,6-trioxospiro(perhydropyrimidino-5)-3'-(2-phenylchromane) (5a).}

Colorless crystals, m.p. $237-238{ }^{\circ} \mathrm{C}$. [Found: $\mathrm{C}, 67.05 ; \mathrm{H}, 4.39$; $\mathrm{N}$, 8.66. $\mathrm{C}_{18} \mathrm{H}_{14} \mathrm{~N}_{2} \mathrm{O}_{4}$ requires $\mathrm{C}$, 67.08; H, 4.38; N, 8.69\%]; $v_{\max }(\mathrm{KBr}) 3197,3087,2850,1692,1491,1432,1367,1258,1200$, $1101,1055,844,749,531,495 \mathrm{~cm}^{-1} ; \delta_{\mathrm{H}}\left(400 \mathrm{MHz}\right.$, DMSO- $\left.d_{6}\right) 11.19(1 \mathrm{H}, \mathrm{s}, \mathrm{N} \underline{\underline{H}}), 11.12(1 \mathrm{H}, \mathrm{s}$, $\mathrm{N \underline {H }}), 7.40(3 \mathrm{H}, \mathrm{m}, \operatorname{Ar} \underline{\mathrm{H}}), 7.22(1 \mathrm{H}, \mathrm{d}, J 8.0 \mathrm{~Hz}, \operatorname{Ar} \underline{\mathrm{H}}), 7.26(2 \mathrm{H}, \mathrm{m}, \operatorname{Ar} \underline{\mathrm{H}}), 7.13(1 \mathrm{H}, \mathrm{t}, J 8.0 \mathrm{~Hz}$, $\operatorname{Ar} \underline{H}), 6.96(1 \mathrm{H}, \mathrm{t}, J 8.4 \mathrm{~Hz}, \operatorname{Ar} \underline{\mathrm{H}}), 6.89(1 \mathrm{H}, \mathrm{d}, J 8.2 \mathrm{~Hz}, \operatorname{Ar} \underline{\mathrm{H}}), 5.08(1 \mathrm{H}, \mathrm{s}, \mathrm{OC} \underline{\mathrm{H}}), 3.24$ and 3.62 $\left(1 \mathrm{H}+1 \mathrm{H}, \mathrm{d}+\mathrm{d}, J 17.0 \mathrm{~Hz}, \mathrm{AB}-\mathrm{system}, \mathrm{CH}_{2} \mathrm{Ar}\right) ; \delta_{\mathrm{C}}\left(100 \mathrm{MHz}\right.$, DMSO- $\left.d_{6}\right) 171.2(\mathrm{O}=\mathrm{C}), 169.3$ $\left(\mathrm{O}=\mathrm{C}^{\prime}\right), 154.4,149.9(\mathrm{O}=\mathrm{C}), 135.4,129.7,129.3,128.7$ (2 C), 127.5 (2 C), 126.9, 121.8, 121.6 , $116.5,81.2,53.1,31.6$.

\subsubsection{1,3-Dimethyl-2,4,6-trioxospiro(perhydropyrimidino-5)-3'-(2-phenylchromane) (5b)}

Colorless crystals, m.p. $161-162{ }^{\circ} \mathrm{C}$. [Found: $\mathrm{C}, 68.45 ; \mathrm{H}, 5.15 ; \mathrm{N}, 7.94 . \mathrm{C}_{20} \mathrm{H}_{18} \mathrm{~N}_{2} \mathrm{O}_{4}$ requires $\mathrm{C}$, 68.56; H, 5.18; N, 8.00\%]; $v_{\max }(\mathrm{KBr}) 3032,2954,1667,1588,1454,1383,1245,1051,920,809$, $755,702,476 \mathrm{~cm}^{-1} ; \delta_{\mathrm{H}}\left(400 \mathrm{MHz}, \mathrm{CDCl}_{3}\right) 7.40(3 \mathrm{H}, \mathrm{m}, \operatorname{Ar} \underline{\underline{H}}), 7.24(4 \mathrm{H}, \mathrm{m}, \operatorname{Ar} \underline{\underline{H}}), 5.13(1 \mathrm{H}, \mathrm{s}$, $\mathrm{OC} \underline{\underline{H}}), 3.14$ and $4.02\left(1 \mathrm{H}+1 \mathrm{H}, \mathrm{d}+\mathrm{d}, J 17.1 \mathrm{~Hz}, \mathrm{AB}\right.$-system, $\left.\underline{\underline{H}}_{2} \mathrm{Ar}\right), 3.09(3 \mathrm{H}, \mathrm{s}, \mathrm{NMe}), 3.06$ ( 3 $\mathrm{H}, \mathrm{s}, \mathrm{NMe}) . \delta_{\mathrm{C}}\left(100 \mathrm{MHz}, \mathrm{CDCl}_{3}\right) 169.0(\mathrm{O}=\mathrm{C}), 167.1(\mathrm{O}=\mathrm{C}), 153.9,150.0(\mathrm{O}=\mathrm{C}), 134.6,129.9$, $128.7(2 \mathrm{C}), 128.4,127.2,126.0,121.9,120.3,116.7,82.0,54.6,31.8,28.6,28.0$.

\subsubsection{2,4,6-Trioxospiro(perhydropyrimidino-5)-3'-(2-phenyl-8-methoxychromane) (5c).}

Colorless crystals, m.p. $309-310{ }^{\circ} \mathrm{C}$. [Found: $\mathrm{C}, 64.74 ; \mathrm{H}, 4.57 ; \mathrm{N}$, 7.93. $\mathrm{C}_{19} \mathrm{H}_{16} \mathrm{~N}_{2} \mathrm{O}_{5}$ requires $\mathrm{C}$, 64.77; H, 4.58; N, 7.95\%]; $v_{\max }(\mathrm{KBr}) 3242,1733,1589,1488,1375,1331,1265,1221,1085,954$, $752,705,522,496 \mathrm{~cm}^{-1} ; \delta_{\mathrm{H}}\left(400 \mathrm{MHz}\right.$, DMSO-d $\left.d_{6}\right) 11.18(1 \mathrm{H}, \mathrm{s}, \mathrm{N} \underline{\mathrm{H}}), 11.12(1 \mathrm{H}, \mathrm{s}, \mathrm{N} \underline{\mathrm{H}}) .7 .41$

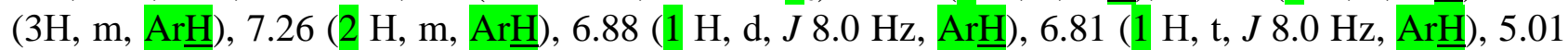
$(1 \mathrm{H}, \mathrm{s}, \mathrm{OC} \underline{\mathrm{H}}), 3.73(3 \mathrm{H}, \mathrm{s}, \mathrm{OMe}), 3.61+3.22\left(1 \mathrm{H}+1 \mathrm{H}, \mathrm{d}+\mathrm{d}, J 17.1 \mathrm{~Hz}, \mathrm{AB}\right.$-system, $\left.\mathrm{CH}_{2} \mathrm{Ar}\right)$; $\delta_{\mathrm{C}}\left(100 \mathrm{MHz}, \mathrm{DMSO}-\mathrm{d}_{6}\right) 171.3(\mathrm{O}=\mathrm{C}), 169.3(\mathrm{O}=\mathrm{C}), 149.9(\mathrm{O}=\mathrm{C}), 148.5,146.0,135.4,129.7$, 128.7 (2 C), 127.5, 122.4, 121.2, $120.8(2 \mathrm{C}), 109.7,80.5,55.9,53.1,31.5$.

\subsubsection{1,3-Dimethyl-2,4,6-trioxospiro(perhydropyrimidino-5)-3'-(2-phenyl-8-methoxychromane)} $(5 d)$.

Colorless crystals, m.p. $170-171^{\circ} \mathrm{C}$. [Found: $\mathrm{C}$, 66.27; $\mathrm{H}, 5.31 ; \mathrm{N}$, 7.31. $\mathrm{C}_{21} \mathrm{H}_{20} \mathrm{~N}_{2} \mathrm{O}_{5}$ requires $\mathrm{C}$, 66.31; H, 5.30; N, 7.36\%]; $v_{\max }(\mathrm{KBr}) 3023,2954,2836,1689,1589,1451,1382,1265,1220$, $1092,896,753,714,612,480 \mathrm{~cm}^{-1} ; \delta_{\mathrm{H}}\left(400 \mathrm{MHz}, \mathrm{CDCl}_{3}\right) 7.37$ (3 H, m, ArH $), 7.24$ (2 H, m, ArH $)$, 6.97 (1 H, t, $J 8.0 \mathrm{~Hz}, \operatorname{Ar} \underline{\mathrm{H}}), 6.81(1 \mathrm{H}, \mathrm{t}, J 8.0 \mathrm{~Hz}, \mathrm{Ar} \underline{\mathrm{H}}), 5.01(1 \mathrm{H}, \mathrm{s}, \mathrm{OC} \underline{\mathrm{H}}), 3.73(3 \mathrm{H}, \mathrm{s}, \mathrm{OMe})$, $3.61+3.22\left(1 \mathrm{H}+1 \mathrm{H}, \mathrm{d}+\mathrm{d}, J 17.1 \mathrm{~Hz}, \mathrm{AB}\right.$-system, $\left.\mathrm{CH}_{2} \mathrm{Ar}\right) ; \delta_{\mathrm{C}}\left(100 \mathrm{MHz}, \mathrm{CDCl}_{3}\right) 171.3(\mathrm{O}=\mathrm{C})$, $169.3\left(\mathrm{O}=C^{4(6)}\right), 149.9(\mathrm{O}=\mathrm{C}), 148.5,144.0,135.4,129.7,128.7(2 \mathrm{C}), 127.5,122.4,121.2,120.8$, 109.7, 80.5, 55.9, 53.1, 31.5.

\subsubsection{2,4,6-Trioxospiro(perhydropyrimidino-5)-3'-(2-(3-chlorophenyl)chromane) (5e).}

Colorless crystals, m.p. $195-196{ }^{\circ} \mathrm{C}$. [Found: $\mathrm{C}, 60.65 ; \mathrm{H}, 3.70 ; \mathrm{N}, 7.84 ; \mathrm{C}_{18} \mathrm{H}_{13}{ }^{35} \mathrm{ClN}_{2} \mathrm{O}_{4}$ requires C, 60.60; H, 3.67; N, 7.85\%]; $v_{\max }(\mathrm{KBr}) 3222,3097,2844,1726,1589,1491,1428,1366,1251$, $1055,757,692,528,500 \mathrm{~cm}^{-1} ; \delta_{\mathrm{H}}\left(400 \mathrm{MHz}\right.$, DMSO- $\left.d_{6}\right) 11.25\left(2 \mathrm{H}, \mathrm{s}, \mathrm{N} \underline{\mathrm{H}}+\mathrm{N}^{\prime} \underline{\underline{H}}\right), 7.45(4 \mathrm{H}, \mathrm{m}$, $\operatorname{Ar} \underline{H}), 7.26(1 \mathrm{H}, \mathrm{d}, J 8.0 \mathrm{~Hz}, \operatorname{Ar} \underline{\mathrm{H}}), 7.13(1 \mathrm{H}, \mathrm{t}, J 8.0 \mathrm{~Hz}, \operatorname{Ar} \underline{H}), 6.98(1 \mathrm{H}, \mathrm{t}, J 8.1 \mathrm{~Hz}, \operatorname{Ar} \underline{\mathrm{H}}), 6.87$ $(1 \mathrm{H}, \mathrm{d}, J 8.1 \mathrm{~Hz}, \mathrm{Ar} \underline{\mathrm{H}}), 5.48(1 \mathrm{H}, \mathrm{s}, \mathrm{OC} \underline{\mathrm{H}}), 3.73+3.23(1 \mathrm{H}+1 \mathrm{H}, \mathrm{d}+\mathrm{d}, J 17.6 \mathrm{~Hz}, \mathrm{AB}-\mathrm{system}$, 
$\left.\mathrm{CH}_{2} \mathrm{Ar}\right) ; \delta_{\mathrm{C}}\left(100 \mathrm{MHz}, \mathrm{DMSO}-d_{6}\right) 169.6(\mathrm{O}=\mathrm{C}), 169.4(\mathrm{O}=\mathrm{C}), 154.2,149.9(\mathrm{O}=\mathrm{C}), 142.0,133.1$, $131.4,129.9,129.6,129.3,127.7,126.9,122.0,121.9,116.4,76.8,52.5,31.4$.

\subsubsection{2,4,6-Trioxospiro(perhydropyrimidino-5)-3'-(2-(2-chlorophenyl)chromane) (5f).}

Colorless crystals, m.p. $159-160{ }^{\circ} \mathrm{C}$. [Found: C, 60.71; H, 3.72; N, 7.77. $\mathrm{C}_{18} \mathrm{H}_{13}{ }^{35} \mathrm{ClN}_{2} \mathrm{O}_{4}$ requires $\mathrm{C}, 60.60 ; \mathrm{H}, 3.67 ; \mathrm{N}, 7.85 \%]$; $v_{\max }(\mathrm{KBr}) 3226,3100,2846,1726,1589,1492,1367,1249,1113$, $754,504 \mathrm{~cm}^{-1} ; \delta_{\mathrm{H}}\left(400 \mathrm{MHz}, \mathrm{DMSO}-d_{6}\right) 11.31(1 \mathrm{H}, \mathrm{s}, \mathrm{NH}), 11.22(1 \mathrm{H}, \mathrm{s}, \mathrm{N} \underline{\mathrm{H}}) .7 .50(1 \mathrm{H}, \mathrm{d}, J 7.9$ $\mathrm{Hz}, \operatorname{Ar} \underline{\mathrm{H}}), 7.45(1 \mathrm{H}, \mathrm{d}, J 7.9 \mathrm{~Hz}, \operatorname{Ar} \underline{\mathrm{H}}), 7.28(1 \mathrm{H}, \mathrm{s}, \operatorname{Ar} \underline{\mathrm{H}}), 7.22(2 \mathrm{H}, \mathrm{m}, \operatorname{Ar} \underline{\mathrm{H}}), 7.14(1 \mathrm{H}, \mathrm{t}, J 8.0$ $\mathrm{Hz}, \operatorname{Ar} \underline{\mathrm{H}}), 6.97$ (1 H, t, J 8.2 Hz, Ar $\underline{\mathrm{H}}), 6.91(1 \mathrm{H}, \mathrm{t}, J 8.2 \mathrm{~Hz}, \operatorname{Ar} \underline{\mathrm{H}}), 5.15(1 \mathrm{H}, \mathrm{s}, \mathrm{OC} \underline{\mathrm{H}}), 3.61+3.25$ $\left(1 \mathrm{H}+1 \mathrm{H}, \mathrm{d}+\mathrm{d}, J 17.7 \mathrm{~Hz}, \mathrm{AB}-\mathrm{system}, \mathrm{CH}_{2} \mathrm{Ar}\right) ; \delta_{\mathrm{C}}\left(100 \mathrm{MHz}, \mathrm{DMSO}-d_{6}\right) 171.0(\mathrm{O}=\mathrm{C}), 169.1$ $(\mathrm{O}=\mathrm{C}), 154.1,149.9(\mathrm{O}=\mathrm{C}), 137.8,130.7,129.7,129.2,127.2,127.0,126.2,121.8,121.6,116.6$, $116.5,82.3,52.9,31.5$.

\subsubsection{2,4,6-Trioxospiro(perhydropyrimidino-5)-3'-(2-(3-methoxyphenyl)chromane) (5g).}

Colorless crystals, m.p. $146-147^{\circ} \mathrm{C}$. [Found: $\mathrm{C}, 64.69 ; \mathrm{H}, 4.60 ; \mathrm{N}, 7.91$, $\mathrm{C}_{19} \mathrm{H}_{16} \mathrm{~N}_{2} \mathrm{O}_{5}$ requires $\mathrm{C}$, 64.77; H, 4.58; N, 7.95\%]; $v_{\max }(\mathrm{KBr}) 3228,3106,1728,1589,1491,1368,1257,1222,1114,1051$, 757, 697, $502 \mathrm{~cm}^{-1} ; \delta_{\mathrm{H}}\left(400 \mathrm{MHz}, \mathrm{DMSO}-d_{6}\right) 11.22(1 \mathrm{H}, \mathrm{s}, \mathrm{N} \underline{\mathrm{H}}), 11.15(1 \mathrm{H}, \mathrm{s}, \mathrm{N} \underline{\mathrm{H}}) .7 .31(1 \mathrm{H}, \mathrm{t}, J$ $8.0 \mathrm{~Hz}, \operatorname{ArH}), 7.22(1 \mathrm{H}, \mathrm{d}, J 8.0 \mathrm{~Hz}, \operatorname{Ar} \underline{\mathrm{H}}), 7.14(1 \mathrm{H}, \mathrm{t}, J 8.0 \mathrm{~Hz}, \operatorname{Ar} \underline{\mathrm{H}}), 6.98(1 \mathrm{H}, \mathrm{d}, J 8.0 \mathrm{~Hz}$, $\operatorname{Ar} \underline{H}), 6.96(1 \mathrm{H}, \mathrm{d}, J 8.0 \mathrm{~Hz}, \operatorname{Ar} \underline{\mathrm{H}}), 6.89(1 \mathrm{H}, \mathrm{t}, J 8.2 \mathrm{~Hz}, \operatorname{Ar} \underline{\mathrm{H}}), 6.83(1 \mathrm{H}, \mathrm{d}, J 8.2 \mathrm{~Hz}, \operatorname{Ar} \underline{\mathrm{H}}), 6.80$ $\left(1 \mathrm{H}, \mathrm{s}, H_{\text {arom }}\right), 5.05(1 \mathrm{H}, \mathrm{s}, \mathrm{OC} \underline{\underline{\mathrm{H}}}), 3.76(3 \mathrm{H}, \mathrm{s}, \mathrm{OMe}), 3.61+3.23(1 \mathrm{H}+1 \mathrm{H}, \mathrm{d}+\mathrm{d}, J 17.7 \mathrm{~Hz}$, AB-system, $\left.\mathrm{CH}_{2} \mathrm{Ar}\right) ; \quad \delta_{\mathrm{C}}\left(100 \mathrm{MHz}, \mathrm{DMSO}-d_{6}\right) 171.2(\mathrm{O}=\mathrm{C}), 169.3(\mathrm{O}=\mathrm{C}), 159.3,154.5,149.9$ $(\mathrm{O}=\mathrm{C}), 136.9,129.9,129.3,127.0,121.7,121.6,119.7,116.5,115.2,113.0,81.1,55.6,53.0,31.6$.

\subsubsection{2,4,6-Trioxospiro(perhydropyrimidino-5)-3'-(2,2-dimethylchromane) (7).}

Colorless crystals, m.p. $314-315^{\circ} \mathrm{C}$. [Found: $\mathrm{C}, 61.28 ; \mathrm{H}, 5.13 ; \mathrm{N}, 10.18 . \mathrm{C}_{14} \mathrm{H}_{14} \mathrm{~N}_{2} \mathrm{O}_{4}$ requires C, 61.31; H, 5.14; N, 10.21\%]; $v_{\max }(\mathrm{KBr}) 3201,3097,1756,1696,1493,1428,1342,1238,1192$, $1144,1116,968,764,544,523 \mathrm{~cm}^{-1} ; \delta_{\mathrm{H}}\left(400 \mathrm{MHz}\right.$, DMSO- $\left.d_{6}\right) 11.37(2 \mathrm{H}, \mathrm{s}, 2 \mathrm{NH}), 7.15(1 \mathrm{H}, \mathrm{d}, J$ $8.0 \mathrm{~Hz}, \operatorname{ArH}), 7.06(1 \mathrm{H}, \mathrm{d}, J 8.0 \mathrm{~Hz}, \operatorname{ArH}), 6.88(1 \mathrm{H}, \mathrm{t}, J 8.0 \mathrm{~Hz}, \operatorname{ArH}), 6.73(1 \mathrm{H}, \mathrm{d}, J 8.0 \mathrm{~Hz}$, $\operatorname{Ar} \underline{\mathrm{H}}), 3.23\left(2 \mathrm{H}, \mathrm{s}, \underline{\mathrm{CH}}_{2}\right) ; 1.31\left(6 \mathrm{H}, \mathrm{s}, \overline{\mathrm{CMe}}_{2}\right) ; \delta_{\mathrm{C}}\left(100 \mathrm{MHz}, \mathrm{DMSO}-d_{6}\right) 170.5(2 \mathrm{O}=\mathrm{C}), 152.3$, $150.8,128.8,126.9,122.4,122.0,116.7,76.7,54.8,27.7 .24 .1$.

4.5. Method $B$ - the procedure for synthesis of 1,3-dimethyl-2,4,6trioxospiro(perhydropyrimidino-5)-3'-(2-phenylchromane) (5b) in presence of succinic acid (Table 1)

1,3-Dimethyl-5-benzylidenebarbituric acid $3 \mathbf{b}(0.35 \mathrm{~g}, 1 \mathrm{mmol})$ was mixed with $0.35 \mathrm{~g}$ of succinic acid and kept under inert atmosphere in a sealed ampoule at $190{ }^{\circ} \mathrm{C}$ for $4 \mathrm{~h}$. After cooling, the product was washed by hot water, dried and extracted by boiling heptane- $\mathrm{CH}_{2} \mathrm{Cl}_{2}(10: 1,3 \times 15$ $\mathrm{mL}$ ). The combined extract was evaporated, the solid residue was washed by hexane and dried in air to give the derivative $\mathbf{3 b}$ as colorless crystals, m.p. $161-162{ }^{\circ} \mathrm{C}$.

4.6. General Method $\mathrm{C}$ - the procedure for synthesis of 5-spiro-3'-chromanes 5c,d and 7 (Table 1) in acetic acid

5-Benzylidene barbituric acid derivative $(\mathbf{3 c}, \mathbf{d}, \mathbf{6 c})(2 \mathrm{mmol})$ was dissolved in acetic acid (10 $\mathrm{mL}$ ) and heated under reflux. The completion of the reaction was established by TLC. After that the solvent was removed under reduced pressure, the residue was washed by aqueous methanol and dried in air. The time of reaction and yield of the obtained 5-spiro-3'-chromane derivatives $(\mathbf{5 c}, \mathbf{d}, \mathbf{7})$ see in Table 1.

4.7. General procedure for synthesis of $2,3,4,6 \mathrm{a}, 7,12 \mathrm{~b}$-hexahydro- $1 \mathrm{H}, 6 \mathrm{H}$ chromeno $\left[4^{\prime}, 3\right.$ ' $\left.: 4,5\right]$ pyrano $[2,3-d]$ pyrimidine-1,3-diones $(9 \mathrm{a}, \mathrm{b})$ 
5-(2-Allyloxybenzylidene)barbituric acid (8a) $(2 \mathrm{mmol})$ or its derivative (8b) were dissolved in acetic acid $(15 \mathrm{~mL})$ and heated under reflux for $12 \mathrm{~h}$. The process was monitored by TLC. After completion of the reaction, the solvent was removed under reduced pressure, the crude residue was washed by methanol and dried in air.

\section{(9a) \\ 4.7.1. 2,3,4,6a,7,12b-hexahydro-1H,6H-chromeno[4',3':4,5]pyrano[2,3-d]pyrimidine-1,3-dione}

Mixture of $\left(R^{*}, S^{*}\right)$ - and $\left(S^{*}, S^{*}\right)$-diastereomers (75\% and $25 \%$, respectively). Colorless crystals, m.p. $360{ }^{\circ} \mathrm{C}$ (decomp.). [Found: $\mathrm{C}, 61.72 ; \mathrm{H}, 4.42 ; \mathrm{N}, 10.28 . \mathrm{C}_{14} \mathrm{H}_{12} \mathrm{~N}_{2} \mathrm{O}_{4}$ requires $\mathrm{C}, 61.76$; $\mathrm{H}$, $4.44 ; \mathrm{N}, 10.29 \%] ; \delta_{\mathrm{H}}\left(400 \mathrm{MHz}, \mathrm{DMSO}-d_{6}\right) 11.23\left(1 \mathrm{H}, \mathrm{s}, \mathrm{NH}\left(\mathrm{S}^{*}, S^{*}+R^{*}, S^{*}\right)\right), 10.78(0.75 \mathrm{H}, \mathrm{s}$, $\left.\mathrm{N} \underline{\mathrm{H}}\left(R^{*}, S^{*}\right)\right), 10.40\left(0.25 \mathrm{H}, \mathrm{s}, \mathrm{N} \underline{\mathrm{H}}\left(S^{*}, S^{*}\right)\right), 7.37+7.29(0.75 \mathrm{H}+0.25 \mathrm{H}, \mathrm{d}+\mathrm{d}, J 8.0 \mathrm{~Hz}, \mathrm{Ar} \underline{\mathrm{H}}$ $\left.\left(R^{*}, S^{*}+S^{*}, S^{*}\right)\right), 7.10+7.01\left(0.25 \mathrm{H}+0.75 \mathrm{H}, \mathrm{t}+\mathrm{t}, J 8.0 \mathrm{~Hz}, \operatorname{Ar} \underline{\mathrm{H}}\left(\mathrm{S}^{*}, S^{*}+R^{*}, S^{*}\right)\right), 6.99+6.78$ $\left(0.25 \mathrm{H}+0.75 \mathrm{H}, \mathrm{t}+\mathrm{t}, J 8.0 \mathrm{~Hz}, \operatorname{Ar} \underline{\mathrm{H}}\left(\mathrm{S}^{*}, S^{*}+R^{*}, S^{*}\right)\right), 6.88+6.65(0.25 \mathrm{H}+0.75 \mathrm{H}, \mathrm{d}+\mathrm{d}, J 8.0$ $\left.\mathrm{Hz}, \operatorname{Ar} \underline{\mathrm{H}}\left(\mathrm{S}^{*}, S^{*}+R^{*}, S^{*}\right)\right), 4.84\left(0.25 \mathrm{H}, \mathrm{s}\right.$ (broad), OC태 $\left(S^{*}, S^{*}\right), 4.54+4.03(0,75 \mathrm{H}+0.75 \mathrm{H}, \mathrm{d}+$ d, $J 11.5 \mathrm{~Hz}, \mathrm{AB}$-system, OC $\left.\underline{\mathrm{H}}_{2}\left(R^{*}, S^{*}\right)\right), 4.49\left(0.25 \mathrm{H}, \mathrm{m}, \mathrm{OC} \underline{\mathrm{H}} \mathrm{H}\left(S^{*}, S^{*}\right)\right), 4.30\left(1.5 \mathrm{H}, \mathrm{OC} \underline{\mathrm{H}}_{2}\right.$ $\left.\left(R^{*}, S^{*}\right)\right), 4.10\left(0.75 \mathrm{H}, \mathrm{d}, J 4.8 \mathrm{~Hz}, \operatorname{ArC} \underline{\mathrm{HCH}}\left(R^{*}, S^{*}\right)\right), 3.75(0.25 \mathrm{H}, \mathrm{d}, J 13.2 \mathrm{~Hz}, \operatorname{ArC} \underline{\mathrm{HCH}}$ $\left.\left(S^{*}, S^{*}\right)\right), 3.74\left(0.25 \mathrm{H}, \mathrm{m}, \mathrm{OCH} \underline{\mathrm{H}}\left(S^{*}, S^{*}\right)\right), 2.37\left(0.75 \mathrm{H}, \mathrm{m}, \mathrm{CH}\left(R^{*}, S^{*}\right)\right), 2.32(0.25 \mathrm{H}, \mathrm{m}, \mathrm{C} \underline{\mathrm{H}}$ $\left.\left(S^{*}, S^{*}\right)\right), 2.11\left(0.25 \mathrm{H}, \mathrm{m}, \mathrm{OCH} H\left(S^{*}, S^{*}\right)\right) ; \delta_{\mathrm{C}}\left(100 \mathrm{MHz}, \mathrm{DMSO}-d_{6}\right) 166.0,163.7,158.7,158.0$, $152.4,150.3,150.2,150.0,136.2,131.4,128.3,128.2,128.1,124.8,124.1,122.0,121.3,116.8$, $89.6,87.9,75.8,73.5,67.1,65.6,32.3,29.3,29.0,27.5$.

\subsubsection{9-methoxy-2,3,4,6a,7,12b-hexahydro-1H,6H-chromeno[4',3':4,5]pyrano[2,3-d]pyrimidine-} 1,3-dione $(\mathbf{9 b})$

Mixture of $\left(R^{*}, S^{*}\right)$ - and $\left(S^{*}, S^{*}\right)$-diastereomers $(77 \%$ and $23 \%$, respectively). Colorless crystals, m.p. $380{ }^{\circ} \mathrm{C}$ (decomp.). [Found: $\mathrm{C}, 59.57 ; \mathrm{H}, 4.65 ; \mathrm{N}, 9.23 . \mathrm{C}_{15} \mathrm{H}_{14} \mathrm{~N}_{2} \mathrm{O}_{5}$ requires $\mathrm{C}, 59.60 ; \mathrm{H}, 4.67$; $\mathrm{N}, 9.27 \%] ; \delta_{\mathrm{H}}\left(400 \mathrm{MHz}, \mathrm{DMSO}-d_{6}\right) 11.41\left(0.77 \mathrm{H}, \mathrm{s}, \mathrm{NH}\left(R^{*}, S^{*}\right)\right), 11.38\left(0.23 \mathrm{H}, \mathrm{s}, \mathrm{N} \underline{\mathrm{H}}\left(S^{*}, S^{*}\right)\right)$, $10.94\left(0.77 \mathrm{H}, \mathrm{s}, \mathrm{NH}\left(R^{*}, S^{*}\right)\right), 10.60\left(0.23 \mathrm{H}, \mathrm{s}, \mathrm{NH}\left(S^{*}, S^{*}\right)\right), 6.73-6.98\left(3 \mathrm{H}, \mathrm{m}, \operatorname{ArH}\left(R^{*}, S^{*}+\right.\right.$

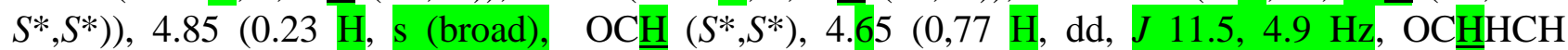
$\left.\left(R^{*}, S^{*}\right)\right), 4.54\left(0.23 \mathrm{H}, \mathrm{dd}, J 13.1,2.8 \mathrm{~Hz}, \mathrm{OCHHCH}\left(S^{*}, S^{*}\right)\right), 4.33+4.25(0,77 \mathrm{H}+0.77 \mathrm{H}, \mathrm{d}+\mathrm{d}$, $J 12.0 \mathrm{~Hz}, \mathrm{AB}$-system, $\left.\mathrm{OCH}_{2}\left(R^{*}, S^{*}\right)\right), 4.07\left(0.77 \mathrm{H}, \mathrm{d}, J 5.2 \mathrm{~Hz}, \operatorname{ArCHCH}\left(R^{*}, S^{*}\right)\right), 3.93(0.77 \mathrm{H}$, m, OCH $\left.\underline{\underline{H}}\left(R^{*}, S^{*}\right)\right), 3.72\left(0.23 \mathrm{H}, \mathrm{m}, \mathrm{OC} \underline{\mathrm{HH}}\left(S^{*}, S^{*}\right)\right), 3.70\left(3 \mathrm{H}, \mathrm{s}, \mathrm{OMe}\left(\mathrm{S}^{*}, S^{*}+R^{*}, S^{*}\right)\right), 3.66$ $\left(0.23 \mathrm{H}, \mathrm{d}, J 13.6 \mathrm{~Hz}, \operatorname{ArC} \underline{\mathrm{HCH}}\left(S^{*}, S^{*}\right)\right), 2.38\left(0.77 \mathrm{H}, \mathrm{m}, \mathrm{C} \underline{\mathrm{H}}\left(R^{*}, S^{*}\right)\right), 2.29(0.23 \mathrm{H}, \mathrm{m}, \mathrm{C} \underline{\mathrm{H}}$ $\left.\left(S^{*}, S^{*}\right)\right), 2.06\left(0.23 \mathrm{H}, \mathrm{m}, \mathrm{OCHH}\left(S^{*}, S^{*}\right)\right) ; \delta_{\mathrm{C}}\left(100 \mathrm{MHz}, \mathrm{DMSO}-d_{6}\right) 166.0,163.7,158.1,158.0$, $152.2,150.3,150.2,148.2,147.6,142.0,137.6,125.2,124.0,122.8,122.1,120.6,111.7,110.5$, $89.6,87.9,76.0,73.3,67.2,65.6,56.1,55.9,32.2,29.2,28.9,27.6$.

\section{Acknowledgements}

This work was supported by the Russian Foundation for Basic Research (grant no. 16-29-10782ofi_m), the Ministry of Education and Science of the Russian Federation (the Agreement number 02.a03.21.0008 of June 24, 2016), and the U.S. National Science Foundation (grant PREM DMR1523611).

\section{Declaration of interest}

The authors of the paper declare no any conflict of interest.

\section{References}

[1] Doran W.J., In: Barbituric acid hypnotics in medicinal chemistry. Blicke FF, Cox RH, editors, vol. IV. Wiley and Sons; New York, 1959, p. 1. 
[2] Vida, J.A.; Gerry, E.H., in Anticonvulsants. Ed. by Vida J.A., New York, Acad. Press 1977, p. 151.

[3] Levina, R.Ya.; Velichko, F.K., Russ. Chem. Rev. (Engl. Transl.) 1960, 29, 427.

[4] Bojarski, J.T.; Mokrosz, J.L.; Bartoń, H.J.; Paluchowska, M.H., Adv. Heterocycl. Chem. 1985, 38, 229.

[5] Krasnov, K.A., in Selected methods for syntheses and modification of heterocycles. Ed by Kartsev V.G., Moscow, IBS Press 2002, vol. 1, p. 280.

[6] Krasnov, K.A., in Selected methods for syntheses and modification of heterocycles. Ed by Kartsev V.G., Moscow, IBS Press 2003, vol. 2, p. 183.

[7] Huang, H.-M.; Procter, D.G., JACS, 2016, 138, 7770.

[8] Platonova, A.Y.; Glukhareva, T.V.; Zimovets, O.A.; Morzherin, Y.Y., Chem. Heterocycl. Comp. 2013, 49, 357.

[9] McQuaid, K.M.; Long, J.Z.; Sames, D., Org. Lett. 2009, 11(14), 2972.

[10] McQuaid, K.M.; Sames, D., JACS. 2009, 131, 402.

[11] Meth-Cohn, O., Adv. Heterocycl. Chem. 1996, 65, 1.

[12] Krasnov, K.A.; Kartsev, V.G., Russ. J. Org. Chem. 2005, 41, 920.

[13] Krasnov, K.A.; Kartsev, V.G.; Khrustalev, V.N., Mendeleev Commun. 2006, 1, 52.

[14] Wang, P.-F.; Jiang, C.-H.; Wen, X.; Xu, Q.-L.; Sun, H., J. Org. Chem. 2015, 80, 1155.

[15] Krasnov, K.A.; Kartsev, V.G.; Khrustalev, V.N. Tetrahedron. 2010. 66, 6054.

[16] Sherry, D.; Thomasco, L.M.; Toogood, P.L. WO Patent 031195, 2004. D'yachenko, E.V.; Glukhareva, T.V.; Nikolaeva, E.F.; Tkachev, A.V.; Morzherin, Yu.Yu., Russ. Chem. Bull. 2004, 6, 1240 .

[17] Tietze, L.F.; Brumby, T.; Pretor, M.; Rember, G., J. Org. Chem. 1988, 53(4), 810.

[18] Evans, P.R., Acta Cryst. 2006, D62, 72.

[19] Sheldrick, G.M. SADABS, v. 2.03, Bruker/Siemens Area Detector Absorption Correction Program; Bruker AXS Inc., Madison, WI, 2003.

[20] Battye, T.G.G.; Kontogiannis, L.; Johnson, O.; Powell, H.R.; Leslie, A.G.W., Acta Cryst. 2011, D67, 271.

[21] Bruker APEX2 software package (Version 1.27). Bruker Molecular Analysis Research Tool. Bruker AXS, Madison, Wisconsin, USA, 2005.

[22] Sheldrick, G.M., Acta Cryst. 2015, C71, 3. 


\section{Graphical Abstract}<smiles>[R]c1cccc(C=C2C(=O)N([Tl])C(=O)N([Tl])C2=O)c1OC([2H])(F)I</smiles>

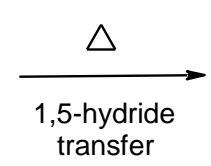<smiles>[R7]c1cccc2c1OC([R])([R])C1(C2)C(=O)N([Tl])C(=O)N([Tl])C1=O</smiles>
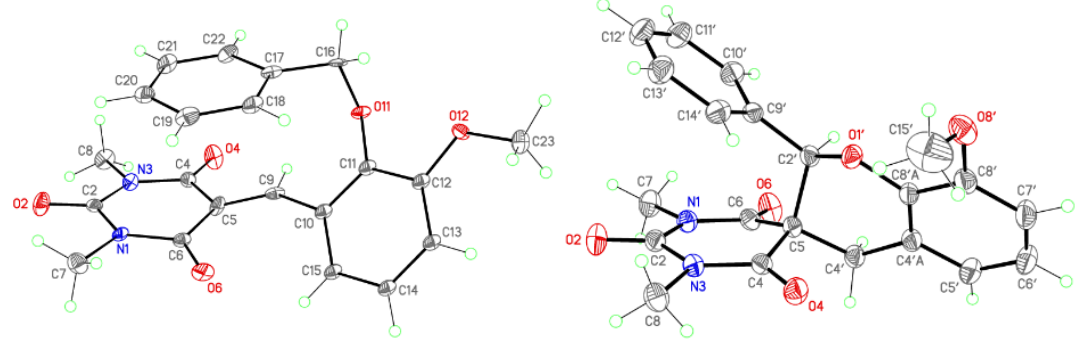

\section{Graphical Abstract}

